\title{
Correlation effects, image charge effects and finite size in the macro-ion-electrolyte system: A field-theoretic approach ${ }^{\star}$
}

\author{
D.J. Lee ${ }^{\mathrm{a}}$ \\ Max-Planck Institut für Physik Komplexer Systeme, Nöthnizer Str. 38, D-01187, Dresden, Germany
}

Received 27 May 2008 and Received in final form 20 October 2008

Published online: 1 May 2009 - (C) EDP Sciences / Società Italiana di Fisica / Springer-Verlag 2009

\begin{abstract}
We consider a model of a macro-ion surrounded by small ions of an electrolyte solution. The finite size of ionic charge distributions of ions, and image charge effects are considered. From such a model it is possible to construct a statistical field theory with a single fluctuating field and derive physical interpretations for both the mean field and two-point correlation function. For point-like charges, at the level of a Gaussian (or saddle point) approximation, we recover the standard Poisson-Boltzmann equation. However, to include ionic correlation effects, as well as image charge effects of individual ions, we must go beyond this. From the field theory considered, it is possible to construct self-consistent approximations. We consider the simplest of these, namely the Hartree approximation. The Hartree equations take the form of two coupled equations. One is a modified Poisson-Boltzmann equation; the other describes both image charge effects on the individual ions, as well as correlations. Such equations are difficult to solve numerically, so we develop an (a WKB-like) approximation for obtaining approximate solutions. This, we apply to a uniformly charged rod in univalent electrolyte solution, for point like ions, as well as for extended spherically symmetric distributions of ionic charge on electrolyte ions. The solutions show how correlation effects and image charge effects modify the Poisson-Boltzmann result. Finite-size charge distributions of the ions reduce both the effects of correlations and image charge effects. For point charges, we test the WKB approximation by calculating a leading-order correction from the exact Hartree result, showing that the WKB-like approximation works reasonably well in describing the full solution to the Hartree equations. From these solutions, we also calculate an effective charge compensation parameter in an analytical formula for the interaction of two charged cylinders.
\end{abstract}

PACS. 61.20.Qg Structure of associated liquids: electrolytes, molten salts, etc. - 61.20.Gy Theory and models of liquid structure -82.35 .Rs Polyelectrolytes

\section{Introduction}

The mechanisms of interaction between cylindrical macroions in electrolyte solutions are still not fully understood [1]. The unmodified PB equation for a uniformly charged cylinder may be inadequate in describing the interaction for a variety of reasons. Correlation effects [1-11], image charge effects $[1,5,8,10,12,13]$, solvent effects $[12,14-19]$, and structure of charged groups at the macro-ion surface $[1,12,19-22]$ may all play an important role. Though, the relative degree of each of these effects is likely to depend on the particular properties of the type of macro-ion under consideration, most importantly its shape, charge density and surface charge distribution of fixed charged groups.

\footnotetext{
* Supplementary material in the form of a doc file available from the journal web page at http://dx/doi/org/10.1140/epje/i2008-10436-x

a e-mail: lee@mpipks-dresden.mpg.de, domolee@hotmail.com
}

Correlation effects may lead to two predicted phenomena, that of charge inversion $[1,7,23,24]$ and samecharge attraction $[1,3,5,7,9,11,25]$. In charge inversion, the amount of positive charge, due to counter-ions, exceeds the surface charge density of the macro-ion effectively making it of opposite charge to its fixed charged groups. Same charge attraction describes a situation in which two macro-ions of the same charge are able to attract each other through interactions with their counterions. Notably, both these phenomena have been predicted in the case of strong correlations between ions at the macro-ion surface $[7,6,25]$, where it is supposed that a Wigner crystal forms on the surface of each macro-ion. Here, the mechanism of attraction is that a Wigner crystal on one macro-ion interlocks with the Wigner crystal on another macro-ion, so that the positive charges associated with one macro-ion lie close to the negative regions on the other and vice versa $[6,25]$. Correlation effects are driven by two main factors: surface charge density and valance 
of counter-ions. As both of these increase, the stronger correlations are expected to become.

In determining correlation effects, both the size and shape of the macro-ion are likely to have an important role. For instance, a flat, infinite charged surface has a proper Wigner crystal phase; but a charged rod may or may not have, depending on its radius. This is owing to the quasi-one-dimensional nature of a cylindrical macroion surface [1], which makes thermal fluctuations at the macro-ion surface, which disrupt such a lattice, much larger.

In a lot of cases, most notably DNA, it may not be sufficient to think of macro-ions as uniformly charged $[1,26]$. It has been shown that the structure of helical macroions, and of counter-ions bound to their surfaces, may cause attraction between them $[1,12,20]$. This mechanism of attraction is most pronounced when ions are able to bind strongly in helical motifs that lie between the helical patterns of fixed charged groups, on the surface of the macro-ion, creating an alternating pattern of charge [1]. In the case of DNA, there is strong evidence to suggest that such localized binding, due to chemi-adsorption, does occur. For instance, evidence suggests this to be the case for polyamines $[1,27,28]$ and $\mathrm{Mn}^{2+}[1,29,30]$, as counter-ions. As in the case of the Wigner crystal, the mechanism of attraction lies in the fact that bound positive charges on one molecule lie close to the negative charges of the other molecule in a commensurate interlocking fashion: an electrostatic zipper [20]. In addition, how the shape of the charge distribution influences the interaction should be important in understanding the formation of cholesteric phases $[31,32]$ and in the statistical mechanics of columnar macro-molecular assemblies [33,34]. Also, there may be reverse interplay between the structure of certain macroions and their interactions; the latter may indeed, sometimes, influence the former $[1,35-37]$. Sill, the parameters that describe the effective charge distributions on helical molecules (KL parameters) [1] have not been calculated from any microscopic theory.

Image charge effects and solvent effects should not be ignored. It seems reasonable to assume that the core of a macro-ion should have a much smaller dielectric response than the surrounding polar solvent [38]. This situation can create repulsive effects due to charges effectively seeing image charge reflections of themselves in regions of low dielectric constant. Even for the interaction between two uniformly charged rods, this can give rise to a new contribution to the interaction potential [12]. This repulsive interaction has half the range of the standard screened electrostatic interaction. It arises from the charges on one molecule experiencing the force due image charges of themselves on the other molecule. Beyond the Poisson-Boltzmann equation, image charge effects in the interaction of individual ions with the small macro-ion may also become important $[5,10]$. Here, the effects could be quite subtle. Image charge effects may increase the threshold at which correlation effects cause attraction, in terms of surface charge density and ionic valance [5]; but, in certain cases, they may cause increased attraction [10]. Other important effects could arise from the discreteness of the solvent. This can yield a non-local and non-linear dielectric response [15-17] and cause hydration forces between macro-ions $[12,14,39]$. Also, one should also point out that image charge effects on solvent molecules could have an important role in determining cylinder-cylinder interactions, at small separations $[40,41]$.

In addition, the finite size of the small ions may have a role in controlling the size of both image charge and correlation effects. As has already been pointed out, in [1], the finite size of charge distributions of certain counter-ions may significantly reduce correlation effects. The saturation of the local density of ions close to the surface of macro-ions [42] should also be considered.

One way of handling some of these effects, in a systematic way, is to reformulate the statistical-mechanical model that describes the macro-ion electrolyte system into a statistical field theory $[3,42,43]$. The advantage in such a formulation is that the mean field of the fluctuating field used in such a theory is proportional to the thermally averaged electrostatic field. This allows for electrostatic boundary conditions due to the macro-ion solvent interface to be handled in a convenient manner. Another possible advantage is that statistical field theories have many levels of self-consistent approximation, the most notable of which are the Hartree approximation and random phase approximations. These self-consistent approximations may be built up in a systematic manner. Such self-consistent approximations have enjoyed notable success in other areas of condensed matter physics, and may yet provide a new useful line of attack in understanding polyelectrolyte interactions. These particular field theories, which arise from such models, enjoy another important advantage: one can do a strong-coupling expansion $[3,5,10]$. Such an expansion is valid when the ions in solution are highly correlated.

Our goal is to follow on from previous work in a series of papers, developing this field-theoretic formulation. We hope to include the effects of finite size, image charge effects, chemi-adsorption, structure of charged groups, and perhaps, later, model the solvent in a more sophisticated way. Though, incorporating the last of these ingredients into the theory is probably the hardest. In this first paper, we start with a single macro-ion in considering some of these effects. We consider a finite-size charge distribution for the small ions and interface effects between the macro-ion and the solvent solution may all be included in a field-theoretic formulation. We then go on to approximate solutions to equations derived from this formalism for the simplest case of a uniformly charged rod.

In Section 2 we start by discussing the statistical mechanical model we shall use. Here, we show how quantities like the average electric field due to the ions and fixed charges, as well as the effective interaction (fluctuating potential) between two (infinitesimal) test charges in solution, may be calculated from the partition function. We also discuss how the more traditional Kirkwood hierarchy [8] is obtained from the model.

Next, we briefly describe how such a model may be transformed into a field theory; the details being left to 
one of the appendices. This is followed by a description of the resulting theory. We also prove both that the average electrostatic potential is indeed proportional to the mean field of the fluctuating field of such a theory, also that the correlation function of the fluctuating field is proportional to the effective interaction between the two test charges. All of this is considered in Section 3.

In Section 4, we describe the Gaussian (saddle-point) approximation of such a field theory. We show that the mean field satisfies the PB equation. Also, we show the form of the free energy, which is not much different from that of [43]. Furthermore, we are able to recast it in a more conventional form [8] and are so able to discuss the physics of each term. In the next section (Sect. 5) we move on to consider the Hartree approximation to our field theory. This takes the form of two coupled equations, not too dissimilar to those derived by the authors of [43] in their most sophisticated variational approximation. However, in our equations, what is new is that the macro-ion solvent interface is fully taken into account with the effects of image charges, as well some finite-size effects of the small ions. Of the derived equations, one is a modified $\mathrm{PB}$ for average electrostatic field, the other an equation for the correlation function. The physics included, beyond the Poisson-Boltzmann formulation, incorporated in such equations is discussed. In Section 6 we are able to extend our formalism to describe extended ionic charges and derive generalized Hartree equations. This completes the formal development.

In Section 7 we solve these equations for the uniformly charged rod, employing a WKB-like approximation to handle the equation for the correlation function. This simplifying approximation is described in the previous two sections. This is similar to the approach used in [13], however this is employed for cylindrical geometry and goes beyond the Gaussian level of approximation. In our calculations we consider only spherically symmetric charge distributions and univalent ions. We start by calculating - for point ions - the average potential, charge density, and a function $\zeta(\mathbf{r})$, which we term the correlation parameter, that describes the relative strength of image and correlation effects on individual ions. To investigate the size of the various effects included in $\zeta(\mathbf{r})$, for some calculations we eliminate some of these effects through simplifying assumptions in regard to the solvent macroion interface. Later we go on to investigate the effects of making the distribution of charge on small ions finite. We see how all of this fits into the framework of an effective theory of interaction of two charged cylinders with image charge effects [12]. Such an effective theory utilizes a Drrjaguin-Landau-Verwey-Overbeek (DLVO) approximation and from our calculations we calculate effective renormalized surface charge densities. Last of all, we estimate the quantitative accuracy of this WKB-like approximation to the exact Hartree solution, by calculating a first-order correction.

At the end we have a discussion of our results (Sect. 8) and a conclusion and outlook (Sect. 9), where we summarize our findings and discuss the extensions that we hope to address in future work.

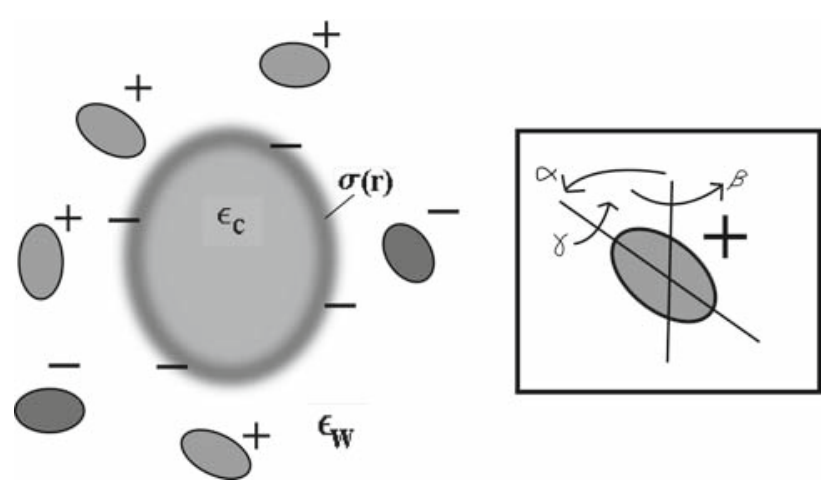

Fig. 1. Schematic picture of the macro-ion-electrolyte system. In the box is shown how the orientation of an ion is described through Euler angles $\boldsymbol{\omega}=(\alpha, \beta, \gamma)$.

\section{The statistical-mechanical model}

\subsection{Description of the model}

Let us consider a statistical-mechanical model of a single large cylindrical macro-ion sitting in a 1:1 electrolyte solution. We center the macro-ion at the origin of a cylindrical coordinate system $(z, R, \varphi)$ so that its major axis lies at $R=0$. As a refinement to [43], we may consider a core region, where $R \leq a$, for which the dielectric constant may be taken to be much lower than the surrounding solvent. We shall consider the case where the dielectric constant of the core is taken to be $\varepsilon_{c} \approx 2$, and that of the surrounding solvent to be $\varepsilon_{w} \approx 80$.

The counter-ions (and electrolyte ions) are restricted to the solvent, which indeed envelops the macro-ion. To enforce this, we may define an ionic exclusion function $\Omega_{I}(\mathbf{r}, \boldsymbol{\omega})$. This function ensures that there is no Boltzmann weight given to the unphysical situation of an ion lying within the core region. If we consider the shape of our small ions as spherical, for a single cylindrical polyelectrolyte of radius $a$ centered at the origin it simply takes the form $\Omega_{I}(\mathbf{r}, \boldsymbol{\omega})=\theta(R-b)$, where $b \geq a$. This inequality accounts for a hard-core radius of each ion. The hard-core radius may be taken to be the radius of the ion together with its inner hydration shell, which is tightly bound to the ion. To study the effects of excluding ions from the macro-ion, sometimes we may relax this requirement and set $\Omega_{I}(\mathbf{r}, \boldsymbol{\omega})=1$.

We suppose that there are only two species of small ion in the solution; one positive and one negative. As a further refinement on reference [43], we start by considering the ionic charge distributions of the small ions to be of arbitrary shape. A schematic picture of the system is shown in Figure 1. Any configuration of the charge of the negative and positive ions may be described through the charge density functions

$$
\hat{\rho}_{+}(\mathbf{r})=\sum_{j=1}^{N_{+}} f_{+}\left(\mathbf{r}-\mathbf{r}_{j}^{+}, \boldsymbol{\omega}_{j}^{+}\right), \quad \hat{\rho}_{-}(\mathbf{r})=\sum_{j=1}^{N_{-}} f_{-}\left(\mathbf{r}-\mathbf{r}_{j}^{-}, \boldsymbol{\omega}_{j}^{-}\right) .
$$


The vectors $\mathbf{r}_{j}^{-}$and $\mathbf{r}_{j}^{+}$describe the positions of negative and positive ions, respectively. The vectors $\boldsymbol{\omega}_{j}^{-}$and $\boldsymbol{\omega}_{j}^{+}$ describe the orientation of each ion and have the three Euler angles $(\alpha, \beta, \gamma)$ as their components. The form factors, $f_{+}$and $f_{-}$describe extended distributions of charge on each ion, and are normalized so that $\int \mathrm{d}^{3} r f(\mathbf{r}, \boldsymbol{\omega})=1$. In the limit of point-like charges, we take $f_{+}\left(\mathbf{r}-\mathbf{r}^{\prime}, \boldsymbol{\omega}\right)=$ $f_{-}\left(\mathbf{r}-\mathbf{r}^{\prime}, \boldsymbol{\omega}\right)=\delta\left(\mathbf{r}-\mathbf{r}^{\prime}\right)$.

For this model we may write down a partition function, in terms of ionic charge density functions

$$
\begin{aligned}
& Z\left[\hat{\rho}_{+}(\mathbf{r}), \hat{\rho}_{-}(\mathbf{r})\right]_{N_{+}, N_{-}}=\frac{1}{N_{+} !} \prod_{j=1}^{N_{+}} \int \frac{\mathrm{d}^{3} r_{j}^{+}}{\lambda_{t}^{3}} \int \frac{\mathrm{d}^{3} \omega_{j}^{+}}{8 \pi^{2}} \\
& \times \frac{1}{N_{-} !} \prod_{j=1}^{N_{-}} \int \frac{\mathrm{d}^{3} r_{j}^{-}}{\lambda_{t}^{3}} \int \frac{\mathrm{d}^{3} \omega_{j}^{-}}{8 \pi^{2}} \\
& \times \Omega_{I}\left(\mathbf{r}_{j}^{+}, \boldsymbol{\omega}_{j}\right) \Omega_{I}\left(\mathbf{r}_{j}^{-}, \boldsymbol{\omega}_{j}\right) \exp \left(-\frac{\tilde{E}_{\mathrm{int}}\left\{\mathbf{r}_{j}, \boldsymbol{\omega}_{j}\right\}}{k_{B} T}\right) \\
& \times \exp \left(-\frac{\tilde{E}_{\mathrm{self}}\left\{\mathbf{r}_{j}, \boldsymbol{\omega}_{j}\right\}}{k_{B} T}\right),
\end{aligned}
$$

where $\lambda_{t}$ is the thermal de Broglie wavelength. Here $N_{+}$ and $N_{-}$are the numbers of small positive and small negative ions, respectively, in the solution.

The total interaction energy between ions $\tilde{E}_{\text {int }}\left\{\mathbf{r}_{j}, \boldsymbol{\omega}_{j}\right\}$, for a given configuration of ions, takes the form (as in [43] for point charges)

$$
\begin{aligned}
& \frac{\tilde{E}_{\mathrm{int}}\left\{\mathbf{r}_{j}, \boldsymbol{\omega}_{j}\right\}}{k_{B} T}=\frac{l_{B}}{2} \int \mathrm{d}^{3} r \int \mathrm{d}^{3} r^{\prime} \\
& \times\left\{\left[q \hat{\rho}_{+}(\mathbf{r})-q \hat{\rho}_{-}(\mathbf{r})+\sigma(\mathbf{r})\right]\left[q \hat{\rho}_{+}\left(\mathbf{r}^{\prime}\right)-q \hat{\rho}_{-}\left(\mathbf{r}^{\prime}\right)+\sigma\left(\mathbf{r}^{\prime}\right)\right]\right. \\
& -q^{2} \sum_{j}\left[f_{-}\left(\mathbf{r}-\mathbf{r}_{j}^{-}, \boldsymbol{\omega}_{j}\right) f_{-}\left(\mathbf{r}^{\prime}-\mathbf{r}_{j}^{-}, \boldsymbol{\omega}_{j}\right)\right. \\
& \left.\left.+f_{+}\left(\mathbf{r}-\mathbf{r}_{j}^{+}, \boldsymbol{\omega}_{j}\right) f_{+}\left(\mathbf{r}^{\prime}-\mathbf{r}_{j}^{+}, \boldsymbol{\omega}_{j}\right)\right]\right\} v\left(\mathbf{r}, \mathbf{r}^{\prime}\right)
\end{aligned}
$$

where $l_{B}=e^{2} /\left(4 \pi \varepsilon_{w} k_{B} T\right)$ is Bjerrum length and $\sigma(\mathbf{r})$ is the charge density of fixed charge groups that lie on the macro-ion. The valance of each ion is denoted by the integer $q$. Since we are only considering a 1:1 electrolyte, both the negative and positive ions have been taken to have the same valance, though the model can be generalized for arbitrary valances. The function eqv $\left(\mathbf{r}, \mathbf{r}^{\prime}\right) / 4 \pi \varepsilon_{w}$ is the electrostatic potential at the point $\mathbf{r}$ due to an ion centered at $\mathbf{r}^{\prime}$, with which the other ions in the system interact. Now, the dielectric boundary, between the solvent and core regions, distorts the electrostatic potential of the ion: it does not take the form of a simple $1 / r$ Coulomb potential. Instead, it must satisfy the following Poisson equation:

$$
-\chi(\mathbf{r}) \nabla^{2} v\left(\mathbf{r}, \mathbf{r}^{\prime}\right)=4 \pi \delta\left(\mathbf{r}-\mathbf{r}^{\prime}\right),
$$

where for a cylindrical macro-ion

$$
\chi(\mathbf{r})=\left[\theta(a-R) \frac{\varepsilon_{c}}{\varepsilon_{w}}+\theta(R-a)\right] .
$$

The usual electrostatic boundary conditions, the continuity of potential and the continuity of the component of the displacement field, D, normal to the dielectric interface, apply at the macro-ion surface. Mathematically, these boundary conditions arise naturally from proper inversion of the operator $\chi(\mathbf{r}) \nabla^{2}$ to find $v\left(\mathbf{r}, \mathbf{r}^{\prime}\right)$. For the moment, we do not actually need to solve this equation for a single ion to make progress.

Now, if we include a region of low dielectric constant, we have a new term in the partition function

$$
\begin{aligned}
& \frac{\tilde{E}_{\text {self }}\left\{\mathbf{r}_{j}, \boldsymbol{\omega}_{j}\right\}}{k_{B} T}=\frac{q^{2} l_{B}}{2} \sum_{j} \int \mathrm{d}^{3} r \int \mathrm{d}^{3} r^{\prime} v\left(\mathbf{r}, \mathbf{r}^{\prime}\right) \\
& \times\left\{\left[f_{-}\left(\mathbf{r}-\mathbf{r}_{j}^{-}, \boldsymbol{\omega}_{j}^{-}\right) f_{-}\left(\mathbf{r}^{\prime}-\mathbf{r}_{j}^{-}, \boldsymbol{\omega}_{j}^{-}\right)\right.\right. \\
& \left.+f_{+}\left(\mathbf{r}-\mathbf{r}_{j}^{+}, \boldsymbol{\omega}_{j}^{+}\right) f_{+}\left(\mathbf{r}^{\prime}-\mathbf{r}_{j}^{-}, \boldsymbol{\omega}_{j}^{+}\right)\right] \\
& -\lim _{r_{j}^{+}, r_{j}^{-} \rightarrow \infty}\left[f_{-}\left(\mathbf{r}-\mathbf{r}_{j}^{-}, \boldsymbol{\omega}_{j}^{-}\right) f_{-}\left(\mathbf{r}^{\prime}-\mathbf{r}_{j}^{-}, \boldsymbol{\omega}_{j}^{-}\right)\right. \\
& \left.\left.+f_{+}\left(\mathbf{r}-\mathbf{r}_{j}^{+}, \boldsymbol{\omega}_{j}^{+}\right) f_{+}\left(\mathbf{r}^{\prime}-\mathbf{r}_{j}^{+}, \boldsymbol{\omega}_{j}^{+}\right)\right]\right\} .
\end{aligned}
$$

This term represents the sum of the changes in the electrostatic self-energy of each ion, moving from the bulk $(\mathbf{r} \rightarrow \infty)$ to a position in the vicinity of the polyelectrolyte, $\mathbf{r}_{j}$. As all ions experience increased repulsion as they move towards the region of low dielectric constant, this term is positive. It depends on the position of each ion; therefore it must be included in the partition function to fully describe the system. Each term in the sum (of eq. (2.6)) may be thought of as a change in hydration energy of a single ion.

\subsection{Mean potential and fluctuation potential}

In such a model we may introduce two test point charges $q_{1}$ and $q_{2}$ lying at the points $\mathbf{r}_{1}$ and $\mathbf{r}_{2}$, respectively. We may define $\tilde{E}_{\text {int }}\left\{\mathbf{r}_{j}, \boldsymbol{\omega}_{j} ; \mathbf{r}_{1}, \mathbf{r}_{2}\right\}$, where we replace $\sigma(\mathbf{r})$ with $\sigma(\mathbf{r})+q_{1} \delta\left(\mathbf{r}-\mathbf{r}_{1}\right)+q_{2} \delta\left(\mathbf{r}-\mathbf{r}_{2}\right)$ in $(2.3)$. These two test charges allow us to calculate two physical quantities

$$
\bar{\phi}\left(\mathbf{r}_{1}\right)=-\lim _{\substack{q_{1} \rightarrow 0 \\ q_{2} \rightarrow 0}}\left[\frac{k_{B} T}{e} \frac{\partial}{\partial q_{1}} \ln Z\left(q_{1}, q_{2}\right)_{N_{+}, N_{-}}\right]
$$

and

$$
W\left(\mathbf{r}_{1}, \mathbf{r}_{2}\right)=-\lim _{\substack{q_{1} \rightarrow 0 \\ q_{2} \rightarrow 0}}\left[k_{B} T \frac{\partial^{2}}{\partial q_{1} \partial q_{2}} \ln Z\left(q_{1}, q_{2}\right)_{N_{+}, N_{-}}\right],
$$

where $Z\left(q_{1}, q_{2}\right)_{N_{+}, N_{-}}$denotes the partition function with the two test charges included. In these definitions, the positions of the test charges are not integrated over. Instead, these charges are taken infinitesimally small so as not to disturb the many-body system. As we will see below, the first of these two expressions is indeed the average potential a test charge experiences from the ions and the fixed charges. Whereas, $q_{1} q_{2} W$ is the average interaction energy between the two test charges. 


$$
\frac{\partial \ln c(\mathbf{r})}{\partial q_{1}}=\frac{\partial \ln Z}{\partial q_{1}}-l_{B} \int \mathrm{d}^{3} r^{\prime} v\left(\mathbf{r}_{1}, \mathbf{r}^{\prime}\right) \frac{\left\langle\left[q \hat{\rho}_{+}\left(\mathbf{r}^{\prime}\right)-q \hat{\rho}_{-}\left(\mathbf{r}^{\prime}\right)+q_{1} \delta\left(\mathbf{r}_{1}-\mathbf{r}^{\prime}\right)+\sigma\left(\mathbf{r}^{\prime}\right)\right]\left[\hat{\rho}_{+}\left(\mathbf{r}_{1}\right)+\hat{\rho}_{-}\left(\mathbf{r}_{1}\right)\right]\right\rangle}{\left\langle\left[\hat{\rho}_{+}\left(\mathbf{r}_{1}\right)+\hat{\rho}_{-}\left(\mathbf{r}_{1}\right)\right]\right\rangle}
$$

Using equations (2.2) and (2.3) in equation (2.7), we may derive the following expressions for $\bar{\phi}$ and $W$ :

$$
\bar{\phi}\left(\mathbf{r}_{1}\right)=\frac{e}{4 \pi \varepsilon_{w}} \int \mathrm{d}^{3} r^{\prime} v\left(\mathbf{r}_{1}, \mathbf{r}^{\prime}\right)\left\langle q \hat{\rho}_{+}\left(\mathbf{r}^{\prime}\right)-q \hat{\rho}_{-}\left(\mathbf{r}^{\prime}\right)+\sigma\left(\mathbf{r}^{\prime}\right)\right\rangle
$$

and

$$
\begin{aligned}
& W\left(\mathbf{r}_{1}, \mathbf{r}_{2}\right)=\frac{e^{2} v\left(\mathbf{r}_{1}, \mathbf{r}_{2}\right)}{4 \pi \varepsilon_{w}} \\
& -\frac{e^{2} l_{B}}{4 \pi \varepsilon_{w}} \int \mathrm{d}^{3} r \int \mathrm{d}^{3} r^{\prime} v\left(\mathbf{r}_{1}, \mathbf{r}\right) v\left(\mathbf{r}_{2}, \mathbf{r}^{\prime}\right) \\
& \times\left[\left\langle\left[q \hat{\rho}_{+}(\mathbf{r})-q \hat{\rho}_{-}(\mathbf{r})\right]\left[q \hat{\rho}_{+}\left(\mathbf{r}^{\prime}\right)-q \hat{\rho}_{-}\left(\mathbf{r}^{\prime}\right)\right]\right\rangle\right. \\
& \left.-\left\langle\left[q \hat{\rho}_{+}(\mathbf{r})-q \hat{\rho}_{-}(\mathbf{r})\right]\right\rangle\left\langle\left[q \hat{\rho}_{+}\left(\mathbf{r}^{\prime}\right)-q \hat{\rho}_{-}\left(\mathbf{r}^{\prime}\right)\right]\right\rangle\right] .
\end{aligned}
$$

Here, $\langle\ldots\rangle$ denotes statistical averaging over all the degrees of freedom associated with the position and orientation of the ions. In equation $(2.8) \int \mathrm{d}^{3} r^{\prime} v\left(\mathbf{r}_{1}, \mathbf{r}^{\prime}\right)\left(q \rho_{+}\left(\mathbf{r}^{\prime}\right)-\right.$ $\left.q \rho_{-}\left(\mathbf{r}^{\prime}\right)+\sigma\left(\mathbf{r}^{\prime}\right)\right)$ is simply the solution of Poisson's equation for each configuration of the ions, which is averaged with the appropriate Boltzmann weight. Therefore, the function $\bar{\phi}\left(\mathbf{r}_{1}\right)$ is, indeed, the mean electrostatic potential of the system.

For equation (2.9) there are two terms. The first term represents the bare electrostatic interaction between the two test charges. The second term is a renormalization of the interaction arising from the movement of the ions to screen out the interaction. We call $W\left(\mathbf{r}_{1}, \mathbf{r}_{2}\right)$ a fluctuating potential. However, this fluctuating potential is quite different from the one given in reference [8]. Here, the latter was only defined for point charges, which we denote by $\phi_{f}\left(q, \mathbf{r}, \mathbf{r}^{\prime}\right)$.

The formula for $\phi_{f}\left(q, \mathbf{r}, \mathbf{r}^{\prime}\right)$ may be found by considering the average density function $c(\mathbf{r})=\left\langle\left[\hat{\rho}_{+}\left(\mathbf{r}_{1}\right)+\hat{\rho}_{-}\left(\mathbf{r}_{1}\right)\right]\right\rangle$, averaged using $\tilde{E}_{\text {int }}\left\{\mathbf{r}_{j} ; q_{1}, 0\right\}$, where the position of the point charge $q_{1}$ is now averaged over and is taken to be finite. The trick is, when $q_{1} \rightarrow q$, it becomes indistinguishable from any small point-like positive ion in the electrolyte solution. Then, one may show that

$$
\text { see equation (2.10) above }
$$

It is possible to get rid of $\frac{\partial \ln Z}{\partial q_{1}}$ by subtracting away the bulk concentration $c_{b}=\lim _{r_{1} \rightarrow \infty}\left\langle\left[\hat{\rho}_{+}\left(\mathbf{r}_{1}\right)+\hat{\rho}_{-}\left(\mathbf{r}_{1}\right)\right]\right\rangle$. In such a way it is possible to show that

$$
\begin{aligned}
\frac{k_{B} T}{e} \frac{\partial}{\partial q_{1}} \ln \left[\frac{c(\mathbf{r})}{c_{b}}\right]= & \bar{\phi}\left(q_{1} ; \mathbf{r}\right)+\phi_{f}\left(q_{1} ; \mathbf{r}, \mathbf{r}\right) \\
& -\lim _{r \rightarrow \infty} \phi_{f}\left(q_{1} ; \mathbf{r}, \mathbf{r}\right),
\end{aligned}
$$

where the fluctuating potential is given by

$$
\begin{aligned}
& \phi_{f}\left(q_{1} ; \mathbf{r}, \mathbf{r}^{\prime}\right)=\frac{e}{4 \pi \varepsilon_{w}} \int \mathrm{d}^{3} r^{\prime \prime} v\left(\mathbf{r}, \mathbf{r}^{\prime \prime}\right) \\
& \times\left[\frac{\left.\left\langle q \hat{\rho}_{+}\left(\mathbf{r}^{\prime \prime}\right)-q \hat{\rho}_{-}\left(\mathbf{r}^{\prime \prime}\right)+q_{1} \delta\left(\mathbf{r}-\mathbf{r}^{\prime \prime}\right)\right]\left[\hat{\rho}_{+}\left(\mathbf{r}^{\prime}\right)+\hat{\rho}_{-}\left(\mathbf{r}^{\prime}\right)\right]\right\rangle}{\left\langle\left[\hat{\rho}_{+}\left(\mathbf{r}^{\prime}\right)+\hat{\rho}_{-}\left(\mathbf{r}^{\prime}\right)\right]\right\rangle}\right. \\
& \left.-\left\langle\left[q \hat{\rho}_{+}\left(\mathbf{r}^{\prime \prime}\right)-q \hat{\rho}_{-}\left(\mathbf{r}^{\prime \prime}\right)\right]\right\rangle\right] .
\end{aligned}
$$

Both equations (2.8) and (2.12) form the starting basis of a hierarchy of equations known as the Kirkwood hierarchy [44]. Evaluation of the concentration is performed by charging the charge $q_{1}$ from 0 up $q$, through integration of equation (2.11) with respect to $q_{1}$. Higher-order equations in the hierarchy may be got by performing successive differentiations in $q_{1}$ on the average number density. Using both equations (2.12) and (2.9), one may relate both fluctuating potentials to one another,

$$
\begin{aligned}
& W\left(\mathbf{r}_{1}, \mathbf{r}_{2}\right)= \\
& l_{B} \int \mathrm{d}^{3} r^{\prime} v\left(\mathbf{r}_{1}, \mathbf{r}^{\prime}\right)\left\langle\hat{\rho}_{+}\left(\mathbf{r}^{\prime}\right)+\hat{\rho}_{-}\left(\mathbf{r}^{\prime}\right)\right\rangle \phi_{f}\left(0 ; \mathbf{r}^{\prime}, \mathbf{r}_{2}\right) \\
& +\frac{e}{4 \pi \varepsilon_{w}} v\left(\mathbf{r}_{1}, \mathbf{r}_{2}\right) .
\end{aligned}
$$

But, as we shall see in the next, the correlation function $W\left(\mathbf{r}_{1}, \mathbf{r}_{2}\right)$ is much more natural for considering correlations in a field-theoretical formulation than $\phi_{f}\left(q ; \mathbf{r}, \mathbf{r}^{\prime}\right)$.

\subsection{Grand canonical ensemble}

In what follows, instead of an ensemble of fixed particle number, it will be more convenient for us to consider the grand partition function, which takes the form

$$
\begin{aligned}
& Z\left[\hat{\rho}_{+}(\mathbf{r}), \hat{\rho}_{-}(\mathbf{r})\right]_{\lambda^{+}, \lambda^{-}}= \\
& \sum_{N_{+}, N_{-}=0}^{\infty}\left(\lambda^{+}\right)^{N_{+}}\left(\lambda^{-}\right)^{N_{-}} Z\left[\hat{\rho}_{+}(\mathbf{r}), \hat{\rho}_{-}(\mathbf{r})\right]_{N^{+}, N^{-}} .
\end{aligned}
$$

Here, $\lambda^{+}$and $\lambda^{-}$are the positive and negative ion fugacities. These may be easily related to usual chemical potentials $\mu_{+}$and $\mu_{-}$simply by $\lambda^{+}=\exp \left(\mu_{+} / k_{B} T\right)$ and $\lambda^{-}=\exp \left(\mu_{-} / k_{B} T\right)$, respectively. The average number of positive ions and negative ions is given by the following expressions:

$$
\left\langle N_{+}\right\rangle=\lambda_{+} \frac{\partial \ln Z_{\lambda}}{\partial \lambda_{+}} \quad \text { and } \quad\left\langle N_{-}\right\rangle=\lambda_{-} \frac{\partial \ln Z_{\lambda}}{\partial \lambda_{-}} .
$$

As the macro-ion-electrolyte system is electrically neutral, we must have the condition that $q\left\langle N_{+}\right\rangle+\int \mathrm{d}^{3} r \sigma(\mathbf{r})=$ $q\left\langle N_{-}\right\rangle$. But, in the thermodynamic limit for an isolated macro-ion in electrolyte solution $\left\langle N_{+}\right\rangle \rightarrow \infty$. Then, for 
point ions and both ions having the same shape, electroneutrality can be achieved by simply setting the two fugacities equal, $\lambda_{+}=\lambda_{-}=\lambda$. Taking account of this, from now on we shall denote the grand canonical ensemble as $Z_{\lambda}$. In the next section we will be able to derive expressions for these quantities in the field-theoretical formulation.

\section{The field-theoretical formulation for point-like ions}

First we consider $f_{+}\left(\mathbf{r}-\mathbf{r}^{\prime}, \boldsymbol{\omega}\right)=f_{-}\left(\mathbf{r}-\mathbf{r}^{\prime}, \boldsymbol{\omega}\right)=\delta\left(\mathbf{r}-\mathbf{r}^{\prime}\right)$. Now, using a trick used in [43], we may start to transform this statistical-mechanical model into a field theory, where we may write the following identity:

$$
\begin{aligned}
& Z\left[\hat{\rho}_{+}(\mathbf{r}), \hat{\rho}_{-}(\mathbf{r})\right]_{\lambda} \equiv \int \mathcal{D} \rho_{+}(\mathbf{r}) \int \mathcal{D} \rho_{-}(\mathbf{r}) \\
& \times \delta^{\infty}\left(\hat{\rho}_{+}(\mathbf{r})-\rho_{+}(\mathbf{r})\right) \delta^{\infty}\left(\hat{\rho}_{-}(\mathbf{r})-\rho_{-}(\mathbf{r})\right) Z\left[\rho_{+}(\mathbf{r}), \rho_{-}(\mathbf{r})\right]_{\lambda} \\
& =\int \mathcal{D} \rho_{+}(\mathbf{r}) \int \mathcal{D} \rho_{-}(\mathbf{r}) \int \mathcal{D} \psi_{+}(\mathbf{r}) \int \mathcal{D} \psi_{-}(\mathbf{r}) \\
& \times \exp \left\{i \int \mathrm { d } ^ { 3 } r \left[\psi_{+}(\mathbf{r})\left(\rho_{+}(\mathbf{r})-\hat{\rho}_{+}(\mathbf{r})\right)\right.\right. \\
& \left.\left.+\psi_{-}(\mathbf{r})\left(\rho_{-}(\mathbf{r})-\hat{\rho}_{-}(\mathbf{r})\right)\right]\right\} \\
& \times Z\left[\rho_{+}(\mathbf{r}), \rho_{-}(\mathbf{r})\right]_{\lambda} .
\end{aligned}
$$

This reformulation (through Eq. (3.1)) has an immediate advantage when we come to consider the grand partition function, as we may use equation (3.1) to readily perform the sums in equation (2.14) (see App. A in Appendices given as supplementary material). As is shown in Appendix A and reference [43], it is possible to integrate out both $\rho_{+}(\mathbf{r})$ and $\rho_{-}(\mathbf{r})$ as well as one of the $\psi$-fields. We integrate out $\psi_{+}(\mathbf{r})$ and set $\psi_{-}(\mathbf{r})=\phi(\mathbf{r})$; in doing so, we arrive at the grand partition function being described by the following field theory:

$$
Z_{\lambda}=\frac{1}{Z_{\mathrm{VAC}}} \int \mathcal{D} \phi(\mathbf{s}) \exp \left(-H_{\mathrm{ion}}[\phi(\mathbf{s})] / \Xi\right),
$$

where

$$
\begin{aligned}
H_{\text {ion }}[\phi(\mathbf{s})]= & \int \mathrm{d}^{3} s\left[\frac{1}{4} \tilde{\chi}(\mathbf{s})(\nabla \phi(\mathbf{s}))^{2}+i \tilde{\sigma}(\mathbf{s}) \phi(\mathbf{s})\right. \\
& \left.-\frac{\tilde{\kappa}^{2}}{2 \Lambda} \tilde{\Omega}_{I}(\mathbf{s}) \cos \phi(\mathbf{s})\right]
\end{aligned}
$$

and $Z\left[\hat{\rho}_{+}(\mathbf{r}), \hat{\rho}_{-}(\mathbf{r})\right]_{\lambda}$ has been simply replaced with $Z_{\lambda}$. Here $\mathbf{s}$ is a rescaled position vector (measured in GouyChapman lengths, so to match with the results of Ref. [43]) so that $\mu \mathbf{s}=\mathbf{r}$, where $\mu=\frac{e}{2 \pi q l_{B} \sigma_{s}}$. We define a new rescaled coordinate system $\left(z_{s}, S, \varphi\right)$. The rescaled functions $\tilde{\chi}(\mathbf{s})$ and $\tilde{\Omega}_{I}(\mathbf{s})$ are defined as $\tilde{\chi}(\mathbf{s})=(\theta(\tilde{a}-$ $\left.S) \varepsilon_{c} / \varepsilon_{w}+\theta(S-\tilde{a})\right)$ and $\tilde{\Omega}_{I}(\mathbf{s}, \boldsymbol{\omega})=\theta(S-\tilde{b})$, respectively, where $\mu \tilde{a}=a$ and $\mu \tilde{b}=b$. Also, in equation (3.3), we have a rescaled charge density so that $\tilde{\sigma}(\mathbf{s})=e \mu \sigma(\mathbf{r}) / \sigma_{s}$. The effective surface charge density $\sigma_{s}$ is given by

$$
\sigma_{s}=e \int \mathrm{d}^{3} r \sigma(\mathbf{r}) / S_{\text {inf }}
$$

and $S_{\text {inf }}$ is the effective surface area of interface. One should note that equation (3.4) is expressed in normal units of length, not Gouy-Chapman lengths, as $\sigma_{s}$ defines the Gouy-Chapman length. If the surface charge density is confined to an infinitesimally thin surface layer, and is uniform about the cylinder, so that $e \sigma(\mathbf{r})=\sigma_{s} \delta(R-a)$, this gives us $\tilde{\sigma}(\mathbf{s})=\delta(S-\tilde{a})$. In this case, $\tilde{\sigma}(\mathbf{s})$ is normalized so that $\int \mathrm{d}^{3} s \tilde{\sigma}(\mathbf{s})=S_{\text {inf }} / \mu^{2} \equiv \tilde{S}_{\text {inf }}$.

Also, we have a rescaled inverse Debye screening length $\tilde{\kappa}=\mu \kappa_{D}$. As usual the Debye screening length for a $1: 1$ electrolyte is given by $\kappa_{D}^{2}=8 \pi q^{2} l_{B} c_{\text {bulk }}$, where $c_{\text {bulk }}$ is the number concentration of ion pairs in the bulk solution. This is related to the rescaled fugacity $\tilde{\lambda}=2 \lambda_{R} / \pi l_{B} \sigma_{s}^{2}$ and $\lambda_{R}=\lambda \exp \left(q^{2} l_{B} v(\infty, \infty) / 2\right) \lambda_{t}^{-3}$, through the following relationship for a single macro-ion:

$$
\tilde{\kappa}^{2}=\tilde{\lambda} \Lambda, \quad \text { where } \quad \Lambda=\lim _{\mathbf{r} \rightarrow \infty}\langle\exp (i \phi(\mathbf{r}))\rangle .
$$

Equation (3.5) is derived in Appendix A.

What plays a crucial role in the theory described by equations $(3.2-3.5)$ is the correlation strength

$$
\Xi=(2 \pi)^{2} l_{B}^{2} \sigma_{s} q^{3} / e .
$$

This is an important measure of the amount of correlations between ions, near the surface of the macro-ion. We will discuss its role in more detail in the next section.

Included in equation (3.2) is

$$
\begin{aligned}
& Z_{\mathrm{VAC}}=\int D \phi(\mathbf{s}) \exp \left[-H_{\mathrm{VAC}}[\phi(\mathbf{s})] / \Xi\right], \\
& H_{\mathrm{VAC}}[\phi(\mathbf{s})]=\int \mathrm{d}^{3} s\left[\frac{1}{4} \chi(\mathbf{s})(\nabla \phi(\mathbf{s}))^{2}\right] .
\end{aligned}
$$

This factor ensures that vacuum fluctuations $(\tilde{\kappa} \rightarrow 0)$ in the field $\phi(\mathbf{s})$ are not considered; the only fluctuations in $\phi(\mathbf{s})$ arise from the movement of the ions.

In the field-theoretical formulation we may evaluate both $\bar{\phi}$ and $W$ for the grand canonical ensemble. We have that

$$
\begin{aligned}
& \bar{\phi}\left(\mathbf{r}_{1}\right)=-\lim _{\substack{q_{1} \rightarrow 0 \\
q_{2} \rightarrow 0}}\left[\frac{k_{B} T}{e} \frac{\partial}{\partial q_{1}} \ln Z_{\lambda}\left(q_{1}, q_{2}\right)\right] \quad \text { and } \\
& W\left(\mathbf{r}_{1}, \mathbf{r}_{2}\right)=-\lim _{\substack{q_{1} \rightarrow 0 \\
q_{2} \rightarrow 0}}\left[k_{B} T \frac{\partial^{2}}{\partial q_{1} \partial q_{2}} \ln Z_{\lambda}\left(q_{1}, q_{2}\right)\right]
\end{aligned}
$$

where one can show

$$
\begin{aligned}
& Z_{\lambda}\left(q_{1}, q_{2}\right)=\frac{1}{Z_{\mathrm{VAC}}} \int \mathcal{D} \phi(\mathbf{s}) \times \\
& \exp \left(-H_{\mathrm{ion}}[\phi(\mathbf{s})] / \Xi-i \frac{q_{1}}{q} \phi\left(\mathbf{s}_{1}\right)-i \frac{q_{2}}{q} \phi\left(\mathbf{s}_{2}\right)\right) .
\end{aligned}
$$


These definitions (cf. Eq. (3.9)) are the same as those of (2.7), except that the quantities are now defined at fixed fugacity and not at fixed particle number. Evaluating both expressions yields the following relationships:

$$
\begin{aligned}
& \bar{\phi}\left(\mathbf{r}_{1}\right)=\frac{i k_{B} T}{q e}\left\langle\phi\left(\mathbf{s}_{1}\right)\right\rangle \quad \text { and } \\
& W\left(\mathbf{r}_{1}, \mathbf{r}_{2}\right) \equiv \frac{k_{B} T}{q^{2}}\left[\left\langle\phi\left(\mathbf{s}_{1}\right) \phi\left(\mathbf{s}_{2}\right)\right\rangle-\left\langle\phi\left(\mathbf{s}_{1}\right)\right\rangle\left\langle\phi\left(\mathbf{s}_{2}\right)\right\rangle\right] \\
& =\frac{k_{B} T \Xi V\left(\mathbf{s}_{1}, \mathbf{s}_{2}\right)}{q^{2}}
\end{aligned}
$$

Note that the functions on the L.H.S. of equation (3.11) are functions of real positions $\mathbf{r}_{1}$ and $\mathbf{r}_{2}$, whereas on the R.H.S. there are functions derived from the field theory of rescaled positions $\mathbf{s}_{1}$ and $\mathbf{s}_{2}$. We indeed find that $q_{1} q_{2} W\left(\mathbf{r}_{1}, \mathbf{r}_{2}\right)$, the average interaction energy between two test charges (at $\mathbf{r}_{1}$ and $\mathbf{r}_{2}$, respectively), is indeed proportional to the correlation function $V\left(\mathbf{s}_{1}, \mathbf{s}_{2}\right)$ and that the mean field is proportional to the electrostatic potential.

Therefore, we have physical interpretations of both the mean field and the two-point correlation function of the field theory.

\section{Gaussian approximation}

Here we evaluate the mean field in the Gaussian approximation. We may write $\phi(\mathbf{s})=\phi^{\prime}(\mathbf{s})-i \tilde{\phi}_{0}(\mathbf{s})$, with $\phi_{0}(\mathbf{s})=i\langle\phi(\mathbf{s})\rangle$. Then we may expand out in powers of the fluctuating field $\phi^{\prime}(\mathbf{s})$. At the lowest order of approximation (the Gaussian) we obtain

$$
\begin{aligned}
Z_{\lambda} \approx & \frac{\exp \left(\left(-H_{0}\left[\phi_{0}(\mathbf{s})\right]+\Lambda_{0} \Delta H\left[\phi_{0}(\mathbf{s})\right]\right) / \Xi\right)}{Z_{\mathrm{VAC}}} \\
& \times \int \mathcal{D} \phi^{\prime}(\mathbf{r}) \exp \left(-H_{G}\left[\phi^{\prime}(\mathbf{s}), \phi_{0}(\mathbf{s})\right] / \Xi\right),
\end{aligned}
$$

where

$$
\begin{aligned}
H_{0}\left[\phi_{0}(\mathbf{s})\right]= & \int \mathrm{d}^{3} s\left[-\frac{1}{4} \chi(\mathbf{s})\left(\nabla \phi_{0}(\mathbf{s})\right)^{2}+\tilde{\sigma}(\mathbf{s}) \phi_{0}(\mathbf{s})\right. \\
& \left.-\frac{\tilde{\kappa}^{2}}{2} \Omega_{I}(\mathbf{s}) \cosh \phi_{0}(\mathbf{s})\right] \\
\Delta H\left[\phi_{0}(\mathbf{s})\right]= & \int \mathrm{d}^{3} s \frac{\tilde{\kappa}^{2}}{2} \Omega_{I}(\mathbf{s}) \cosh \phi_{0}(\mathbf{s}), \\
H_{G}\left[\phi^{\prime}(\mathbf{s}), \phi_{0}(\mathbf{s})\right]= & \int \mathrm{d}^{3} s\left[\frac{1}{4} \chi(\mathbf{s})\left(\nabla \phi^{\prime}(\mathbf{s})\right)^{2}\right. \\
& \left.+\frac{\tilde{\kappa}^{2}}{4} \Omega_{I}(\mathbf{s}) \phi^{\prime}(\mathbf{s})^{2} \cosh \phi_{0}(\mathbf{s})\right]
\end{aligned}
$$

and $\Lambda_{0}=1 / 2 \lim _{r \rightarrow \infty}\left\langle\phi^{\prime}(\mathbf{s})^{2}\right\rangle$. The mean field must satisfy the condition $\delta H_{0}\left[\phi_{0}(\mathbf{s})\right] / \delta \phi_{0}(\mathbf{s})=0$, because we require that $\left\langle\phi^{\prime}(\mathbf{s})\right\rangle=0$. This yields

$$
\chi(\mathbf{s}) \nabla^{2} \phi_{0}(\mathbf{s})-\tilde{\kappa}^{2} \Omega_{I}(\mathbf{s}) \sinh \left(\phi_{0}(\mathbf{s})\right)=-2 \sigma(\mathbf{s}) .
$$

This is simply the non-linear PB equation. The free energy is given by

$$
\begin{aligned}
F= & \frac{k_{B} T}{\Xi} H_{0}\left[\phi_{0}(\mathbf{s})\right]-\frac{\Lambda_{0} k_{B} T}{\Xi} \Delta H\left[\phi_{0}(\mathbf{s})\right] \\
& -k_{B} T \ln \int \mathcal{D} \phi^{\prime}(\mathbf{s}) \exp \left(-H_{G}\left[\phi^{\prime}(\mathbf{s}), \phi_{0}(\mathbf{s})\right] / \Xi\right) \\
& +k_{B} T \ln Z_{\mathrm{VAC} .}
\end{aligned}
$$

The sum of the last three terms may be considered as the leading-order contribution due correlation effects. The last term in equation (4.6) removes a divergence present in the second to last term, due to vacuum fluctuations, which are not considered in the theory. This free energy (Eq. (4.6)) has been considered for the case of two charged plates $[10,13]$. This result is the same as the one given in [43] (for the simpler variational principle of a renormalized charge density, see therein) when there is no dielectric boundary (i.e. setting $\varepsilon_{w}=\varepsilon_{c}$ ), allowing ions to penetrate into the macro-ion, and replacing $\cosh \phi_{0}(\mathbf{s})$ with 1 in both equation (4.3) and equation (4.4) Following this prescription, we indeed recover the expression given by (31) of [43], by rewriting equation (4.5)

$$
\nabla^{2} \phi_{0}(\mathbf{s})=-\tilde{\sigma}(\mathbf{s})-\tilde{\sigma}_{C}(\mathbf{s})=-2 \eta(\mathbf{s}) \tilde{\sigma}(\mathbf{s})-\tilde{\kappa}^{2} \phi_{0}(\mathbf{s}) .
$$

In the limit of no correlations $\Xi \rightarrow 0$ the last two terms may be neglected in equation (4.6). By correctly identifying entropic and electrostatic energy components, it is possible to write the free energy, in the limit $\Xi \rightarrow 0$, in a more conventional form [8]

$$
\begin{aligned}
F= & \frac{e^{2}}{2} \int \mathrm{d}^{3} r \bar{\phi}(\mathbf{r})\left[n_{+}(\mathbf{r})-n_{-}(\mathbf{r})+\sigma(\mathbf{r})\right] \\
& -k_{B} T \int \mathrm{d}^{3} r\left[n_{+}(\mathbf{r}) \ln n_{+}(\mathbf{r})+n_{-}(\mathbf{r}) \ln n_{-}(\mathbf{r})\right] \\
& +k_{B} T \int \mathrm{d}^{3} r\left[n_{+}(\mathbf{r})+n_{-}(\mathbf{r})\right]
\end{aligned}
$$

where $n_{+}(\mathbf{r})$ and $n_{-}(\mathbf{r})$ are the mean number densities of positive and negative ions, respectively.

The first term in equation (4.8) is the electrostatic part, and the second term is the contribution from the entropies of positive and negative ions. Here the last term in equation (4.8) is the contribution from entropy of the solvent for an ideal solution

$$
\begin{aligned}
S_{\text {solv }}= & -\frac{k_{B}}{V_{\text {ion }}} \int \mathrm{d}^{3} r\left[1-V_{\text {ion }}\left(n_{+}(\mathbf{r})+n_{-}(\mathbf{r})\right)\right] \\
& \times \ln \left(1-V_{\text {ion }}\left(n_{+}(\mathbf{r})+n_{-}(\mathbf{r})\right)\right),
\end{aligned}
$$

expanded out for small $n_{+}(\mathbf{r})$ and $n_{-}(\mathbf{r})$. Here $V_{\text {ion }}$ is the volume occupied by one single ion. If we choose not to expand $n_{+}(\mathbf{r})$ and $n_{+}(\mathbf{r})$ in equation (4.9) and minimize the resulting free energy with respect to $n_{+}(\mathbf{r})$ and $n_{-}(\mathbf{r})$, subject to Poisson's equation $\nabla^{2} \phi(\mathbf{r})=-e\left[q n_{+}(\mathbf{r})-q n_{-}(\mathbf{r})+\right.$ $\sigma(\mathbf{r})]$, we arrive at the equations for finite-size ions effects discussed in reference [42]. 
We may ask what is the physical meaning of the last two terms in equation (4.6). First, one is able to show that

$$
\begin{aligned}
\tilde{F} & \equiv k_{B} T \ln \int \mathcal{D} \phi^{\prime}(\mathbf{s}) \exp \left(-H_{G}\left[\phi^{\prime}(\mathbf{s}), \phi_{0}(\mathbf{s})\right] / \Xi\right)-\ln Z_{\mathrm{VAC}} \\
& =-\frac{k_{B} T}{4 \Xi} \int_{0}^{\tilde{\kappa}^{2}} \mathrm{~d} \tilde{\kappa}^{\prime 2} \int \mathrm{d}^{3} s \Omega_{I}(\mathbf{s}) \frac{\partial\left(\tilde{\kappa}^{\prime 2} \cos \phi_{0}(\mathbf{s})\right)}{\partial \tilde{\kappa}^{\prime 2}}\left\langle\phi^{\prime}(\mathbf{s})^{2}\right\rangle \\
& =-q^{2} \int_{0}^{c_{\text {bulk }}} \mathrm{d} c \int \mathrm{d}^{3} r\left(\frac{\partial n_{+}(\mathbf{r} ; c)}{\partial c}+\frac{\partial n_{-}(\mathbf{r} ; c)}{\partial c}\right) W(\mathbf{r}, \mathbf{r} ; c) .
\end{aligned}
$$

Here, bulk concentration is given by $c_{\text {bulk }}$. The physical meaning of this term is quite clear if we consider a small change from a bulk concentration $c_{\text {bulk }}$ to a concentration $c_{\text {bulk }}+\Delta c_{\text {bulk }}$. The small change in this energy term is given by

$$
\begin{aligned}
\Delta \tilde{F}= & -q^{2} \int \mathrm{d}^{3} r\left(n_{+}\left(\mathbf{r} ; c_{\text {bulk }}+\Delta c_{\text {bulk }}\right)-n_{+}\left(\mathbf{r} ; c_{\text {bulk }}\right)\right. \\
& +n_{-}\left(\mathbf{r} ; c_{\text {bulk }}+\Delta c_{\text {bulk }}\right)-n_{-}\left(\mathbf{r} ; c_{\text {bulk }}\right) \\
& \left.\times W\left(\mathbf{r}, \mathbf{r} ; c_{\text {bulk }}+\Delta c_{\text {bulk }}\right)\right) .
\end{aligned}
$$

It is easy to interpret this term as the sum of selfenergies or change in solvation energy of each of the ions when changing the concentration by an infinitesimal amount. This is indeed, simply, the self energy of each ion $q^{2} W\left(\mathbf{r}, \mathbf{r} ; c_{\text {bulk }}\right)$ integrated with the change in the local electrolyte density $\partial\left(n_{+}(\mathbf{r} ; c)+n_{-}(\mathbf{r} ; c)\right) / \partial c$ with concentration. Since both the number densities and self-energies must change with concentration, to calculate the selfenergy contribution, from all the ions to the free energy, one must consider equation (4.10). Whereas, the change in the solvation energy of a single ion going from a region with salt concentration $c_{\text {bulk }}^{\prime}$ to $c_{\text {bulk }}$ would be simply $q^{2}\left(W\left(\mathbf{r}, \mathbf{r} ; c_{\text {bulk }}^{\prime}\right)-W\left(\mathbf{r}, \mathbf{r} ; c_{\text {bulk }}\right)\right)$, provided that this does not effect the concentration of the two regions. The term proportional to $\Lambda_{0}$ simply removes a divergence in equation (4.11) due to the point-like nature of the ions.

Thus, we see that the field-theoretical formulation correctly describes the physics at this level of approximation. But, the Gaussian approximation is only valid provided that $\tilde{\kappa}$ and $\Xi$ remain small. A perturbation theory may be developed for corrections to the PB in powers of $\Xi$ (for details see App. B). But, to consider larger values of $\Xi$, it is possible to use a self-consistent approximation. We do so in the next section.

\section{The Hartree approximation}

We now go beyond the Gaussian approximation to include counter-ion correlation effects. We do this as in [43], and construct a variational trail energy functional, describing the fluctuating part of the field, of the form

$$
H_{H}\left[\phi^{\prime}(\mathbf{s})\right]=\int \mathrm{d}^{3} r \phi^{\prime}(\mathbf{s}) V_{H}\left(\mathbf{s}, \mathbf{s}^{\prime}\right) \phi^{\prime}\left(\mathbf{s}^{\prime}\right) .
$$

We then may expand the partition function about

$$
Z_{H}\left[\phi^{\prime}(\mathbf{s})\right]=\int \mathcal{D} \phi^{\prime}(\mathbf{s}) \exp \left(-H_{H}\left[\phi^{\prime}(\mathbf{s})\right] / \Xi\right)
$$

to first order in $H_{\text {ion }}[\phi(\mathbf{r})]-H_{H}\left[\phi^{\prime}(\mathbf{r})\right]$. Then, we have the following expression for the free energy:

$f_{H}=F_{H} / k_{B} T=-\ln Z_{H}+\frac{1}{\Xi}\left\langle H_{\text {ion }}[\phi(\mathbf{s})]-H_{H}\left[\phi^{\prime}(\mathbf{s})\right]\right\rangle_{H}$.

The subscript $H$ on the averaging bracket denotes averaging with the Boltzmann weight $\exp \left(-H_{H}\left[\phi^{\prime}(\mathbf{s})\right] / \Xi\right)$. On evaluation of the various terms, equation (5.3) yields the following reduced free energy:

$$
\begin{aligned}
f_{H}= & -\frac{1}{2} \operatorname{Tr} \ln V_{H}\left(\mathbf{s}, \mathbf{s}^{\prime}\right)-\frac{1}{\Xi} \int \mathrm{d}^{3} s\left[\left\{\tilde{\chi}(\mathbf{s}) \frac{\left(\nabla \phi_{0}(\mathbf{s})\right)^{2}}{4}\right.\right. \\
& \left.\left.+\tilde{\sigma}(\mathbf{s}) \phi_{0}(\mathbf{s})+\frac{\tilde{\kappa}^{2}}{2} \tilde{\Omega}_{I}(\mathbf{s}) \zeta(\mathbf{s}) \cosh \phi_{0}(\mathbf{s})\right\}\right] \\
& +\frac{1}{4} \int \mathrm{d}^{3} s \int \mathrm{d}^{3} s^{\prime} \delta\left(\mathbf{s}-\mathbf{s}^{\prime}\right) \tilde{\chi}(\mathbf{s}) \nabla_{\mathbf{s}} \nabla_{\mathbf{s}^{\prime}} V_{H}\left(\mathbf{s}, \mathbf{s}^{\prime}\right) \\
& -\frac{1}{2} \int \mathrm{d}^{3} s \int \mathrm{d}^{3} s^{\prime} \delta(0),
\end{aligned}
$$

where

$$
\zeta(\mathbf{s})=\exp \left(-\Xi\left(V_{H}(\mathbf{s}, \mathbf{s})-V_{H}(\infty, \infty)\right) / 2\right) .
$$

The quantity $\zeta(\mathbf{s})$ is essentially a Boltzmann weight associated with the change in the ionic self-energy going from the Bulk to a rescaled position s near the macro-ion.

We may use $f_{H}$ to construct a variational principle as it satisfies the Gibbs-Bogoliubov inequality. This states that $f_{H} \geq f$, where $f$ is the exact reduced free energy. For such a principle, we may treat $V_{H}\left(\mathbf{s}, \mathbf{s}^{\prime}\right)$ and $\phi_{0}(\mathbf{s})$ as variational parameters to minimize the free energy, i.e.,

$$
\delta f_{H} / \delta V_{H}\left(\mathbf{s}, \mathbf{s}^{\prime}\right)=0 \quad \text { and } \quad \delta f_{H} / \delta \phi_{0}(\mathbf{s})=0 .
$$

This yields the Hartree equations

$$
\begin{aligned}
& \tilde{\chi}(\mathbf{s}) \nabla^{2} \phi_{0}(\mathbf{s})-\tilde{\Omega}_{I}(\mathbf{s}) \tilde{\kappa}^{2} \zeta(\mathbf{s}) \sinh \phi_{0}(\mathbf{s})=-2 \tilde{\sigma}(\mathbf{s}), \\
& \tilde{\chi}(\mathbf{s}) \nabla_{\mathbf{s}}^{2} V_{H}\left(\mathbf{s}, \mathbf{s}^{\prime}\right)-\tilde{\Omega}_{I}(\mathbf{s}) \tilde{\kappa}^{2} \zeta(\mathbf{s}) \cosh \phi_{0}(\mathbf{s}) V_{H}\left(\mathbf{s}, \mathbf{s}^{\prime}\right)= \\
& -2 \delta\left(\mathbf{s}^{\prime}-\mathbf{s}\right) .
\end{aligned}
$$

Such an approximation may, indeed, be called a Hartree approximation, as these same equations may also be derived by diagrammatic expansion, where one sums over a particular class of diagrams that correspond to such an approximation in field theory [45]. This diagrammatic derivation is shown in Appendix B.

If we set $\zeta(\mathbf{s})=1$ in equation (5.8), then $V_{H}\left(\mathbf{s}, \mathbf{s}^{\prime}\right)$ obeys the same equation as $\phi_{f}\left(q, \mathbf{r}^{\prime}, \mathbf{r}\right)$ (except for rescaling) in the linearized approximation described in [8]. In such an approximation one can show that $\phi_{f}\left(q, \mathbf{r}^{\prime}, \mathbf{r}\right)$ is linear in $q$. It is, then, not hard to show, under this prescription, that both approximations yield the same Boltzmann weight $\zeta(\mathbf{s})$ and so the same modified PB equation. However, equation (5.8) with $\zeta(\mathbf{s}) \neq 1$ both formulations yield different equations. We suggest that the Hartree approximation may be more physically appropriate, as it depends self-consistently on the local density of ions (see discussion 
at the end of the section), which may be shown to take the form $c(\mathbf{s})=\tilde{\Omega}_{I}(\mathbf{s}) \tilde{\kappa}^{2} \zeta(\mathbf{s}) \cosh \phi_{0}(\mathbf{s})$.

If in equations (5.7) and (5.8) we take the limit $\Xi \rightarrow 0$, we get results for both $\phi_{0}(\mathbf{s})$ and $V\left(\mathbf{s}, \mathbf{s}^{\prime}\right)$ in the Gaussian approximation and these equations become exact. These equations also incorporate completely the first-order correction due to perturbation theory (see App. B). Therefore, for small $\Xi$, they are indeed expected to give solutions close to the first-order perturbation result. However, up to what $\Xi$ the Hartree approximation remains valid has yet to be seen.

In general we cannot obtain an analytical solution to equation (5.8) and the coupled equations are difficult to solve numerically as they stand. However in Appendix C, we are able to construct an approximate solution, which is used in the limit $\mathbf{s}^{\prime} \rightarrow \mathbf{s}$ to calculate $\zeta(\mathbf{s})$ self-consistently. Instead of solving equation (5.8) we solve

$$
\begin{aligned}
& \tilde{\chi}(\mathbf{s}) \nabla_{\mathbf{s}}^{2} \tilde{V}_{H}\left(\mathbf{s}, \mathbf{s}^{\prime}\right)-\tilde{\Omega}_{I}(\mathbf{s}) \tilde{\kappa}^{2} \zeta\left(\mathbf{s}^{\prime}\right) \cosh \phi_{0}\left(\mathbf{s}^{\prime}\right) \tilde{V}_{H}\left(\mathbf{s}, \mathbf{s}^{\prime}\right)= \\
& -2 \delta\left(\mathbf{s}^{\prime}-\mathbf{s}\right) .
\end{aligned}
$$

In equation (5.9) we have replaced $\zeta(\mathbf{s}) \cosh \phi_{0}(\mathbf{s})$ with $\zeta\left(\mathbf{s}^{\prime}\right) \cosh \phi_{0}\left(\mathbf{s}^{\prime}\right)$. We call this a WKB-like approximation. Like the WKB approximation for a Green's function described by equation (5.8), its accuracy depends on how quickly $\tilde{\kappa}^{2} \zeta(\mathbf{s}) \cosh \phi_{0}(\mathbf{s})$ varies. In Appendix C, we show how one may calculate the difference $\Delta V_{H}\left(\mathbf{s}, \mathbf{s}^{\prime}\right)=$ $V_{H}\left(\mathbf{s}, \mathbf{s}^{\prime}\right)-\tilde{V}_{H}\left(\mathbf{s}, \mathbf{s}^{\prime}\right)$ and so capture the exact Hartree result in the form of an integral equation, which may be easier to work with when obtaining exact solutions to equations (5.7) and (5.8). We utilize the expression for $\Delta V_{H}\left(\mathbf{s}, \mathbf{s}^{\prime}\right)$ to calculate a leading-order correction to the solution of equation (5.9). Provided that the size of the correction remains small, the solution to equation (5.9) captures all the important qualitative physics (explained below).

We obtain a partial solution to (5.9) (see App. C) of the following form:

$$
\tilde{V}_{H}(\mathbf{s}, \mathbf{s})-\tilde{V}_{H}(\infty, \infty)=\tilde{V}_{H g}(\mathbf{s}, \mathbf{s})+\delta V_{I}(\mathbf{s}, \mathbf{s}),
$$

where

$$
\delta V_{I}(\mathbf{s}, \mathbf{s})=\frac{\tilde{\kappa}-\hat{\kappa}(\mathbf{s}, 0)}{2 \pi}
$$

and

$$
\hat{\kappa}(\mathbf{s}, \tilde{q})=\sqrt{\tilde{\kappa}^{2} \zeta(\mathbf{s}) \cos \phi_{0}(\mathbf{s})+\tilde{q}^{2}} .
$$

Here, $\tilde{V}_{H g}(\mathbf{s}, \mathbf{s})$ arises from the interface. If we were to allow for ions to penetrate into the macro-ion, we would set $\tilde{\Omega}_{I}(\mathbf{s})=1$, and allow the dielectric to be uniform so that $\tilde{\chi}(\mathbf{s})=1$. The upshot is to cause $\tilde{V}_{H g}(\mathbf{s}, \mathbf{s})=0$. For
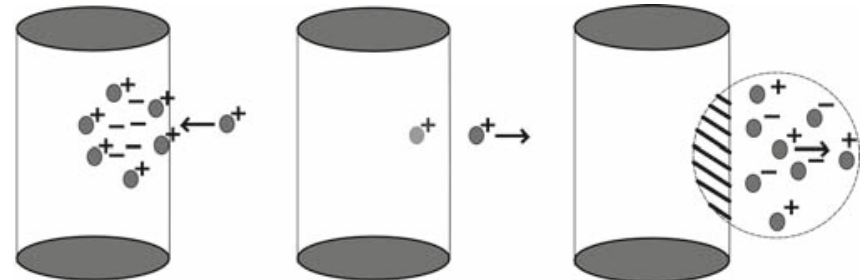

Fig. 2. Schematic drawings of correlation effects (left), image charge effects (center), and exclusion of ions (right). In the first (from the left), ions adjust to the field of an ion creating a correlation hole which draws the ion closer to the macroion. In the second, an ion sees its image charge reflection at the dielectric boundary, from which it is repelled. In the third, part of the Debye atmosphere (shown schematically as a circle with a positive ion at the center) is lost due the exclusion of ions from the macro-ion. The hatched area refers to part of the Debye atmosphere that is lost.

$$
\begin{aligned}
& \tilde{V}_{H g}(\mathbf{s}, \mathbf{s}) \text { we obtain (App. C) } \\
& \tilde{V}_{H g}(\mathbf{s}, \mathbf{s})= \\
& \quad-\frac{2}{(2 \pi)^{2}} \sum_{n=-\infty}^{\infty} \int_{-\infty}^{\infty} \mathrm{d} \tilde{q} K_{n}(\hat{\kappa}(\mathbf{s}, \tilde{q}) S) K_{n}(\hat{\kappa}(\mathbf{s}, \tilde{q}) S) \\
& \times\left[\hat{\kappa}(\mathbf{s}, \tilde{q}) I_{n}^{\prime}(\hat{\kappa}(\mathbf{s}, \tilde{q}) \tilde{b})\left(I_{n}(\tilde{q} \tilde{b})+\xi_{n}(\tilde{a}, \tilde{q}) K_{n}(\tilde{q} \tilde{b})\right)\right. \\
& \left.-q I_{n}(\hat{\kappa}(\mathbf{s}, \tilde{q}) \tilde{b})\left(I_{n}^{\prime}(\tilde{q} \tilde{b})+\xi_{n}(\tilde{a}, \tilde{q}) K_{n}^{\prime}(\tilde{q} \tilde{b})\right)\right] \\
& \quad \times\left[\hat{\kappa}(\mathbf{s}, \tilde{q}) K_{n}^{\prime}(\hat{\kappa}(\mathbf{s}, \tilde{q}) \tilde{b})\left(I_{n}(\tilde{q} \tilde{b})+\xi_{n}(\tilde{a}, \tilde{q}) K_{n}(\tilde{q} \tilde{b})\right)\right. \\
& \left.-q K_{n}(\hat{\kappa}(\mathbf{s}, \tilde{q}) \tilde{b})\left(I_{n}^{\prime}(\tilde{q} \tilde{b})+\xi_{n}(\tilde{a}, \tilde{q}) K_{n}^{\prime}(\tilde{q} \tilde{b})\right)\right]^{-1}
\end{aligned}
$$

where

$$
\xi_{n}(\tilde{a}, \tilde{q})=\frac{(\gamma-1) I_{n}^{\prime}(\tilde{q} \tilde{a}) I_{n}(\tilde{q} \tilde{a})}{\left(K_{n}^{\prime}(\tilde{q} \tilde{a}) I_{n}(\tilde{q} \tilde{a})-\gamma K_{n}(\tilde{q} \tilde{a}) I_{n}^{\prime}(\tilde{q} \tilde{a})\right)}
$$

and $\gamma=\varepsilon_{c} / \varepsilon_{w}$.

Now let us discuss each of the physics included in equations (5.10-5.14). Firstly, equation (5.11) represents a change in hydration energy of an ion moving from a bulk (rescaled) concentration $\tilde{\kappa}^{2}$ to a (rescaled) local concentration $\hat{\kappa}(\mathbf{s}, 0)^{2}$. If $\hat{\kappa}(\mathbf{s}, 0)>\tilde{\kappa}$, this term is negative. Then, when an ion moves towards the macro-ion, its overall potential energy is reduced due to neighboring ions being correlated with it. These ions adjust themselves in the field of the ion, essentially creating a correlation hole [46], see Figure 2. This effect should, indeed, depend on the local density of ions, and become more pronounced when this increases as more ions can adjust themselves to the field of a single ion.

Next, there is an effect due to the exclusion of ions. In Figure 3a we plot $\tilde{V}_{H g}(\mathbf{s}, \mathbf{s})$ as a function of $\gamma$ and fixed $\hat{\kappa}$ at $S=5.91$. We see that as we increase $\hat{\kappa}$, and so increase the local density of ions, we see that $\tilde{V}_{H g}(\mathbf{s}, \mathbf{s})$ increases. As no ions may penetrate the core, the core region cannot participate in screening out the electrostatic self-energy of an ion. So, this hole in the Debye atmosphere about an ion provides a positive contribution to $V_{H}(\mathbf{s}, \mathbf{s})-V_{H}(\infty, \infty)$ 
a.)

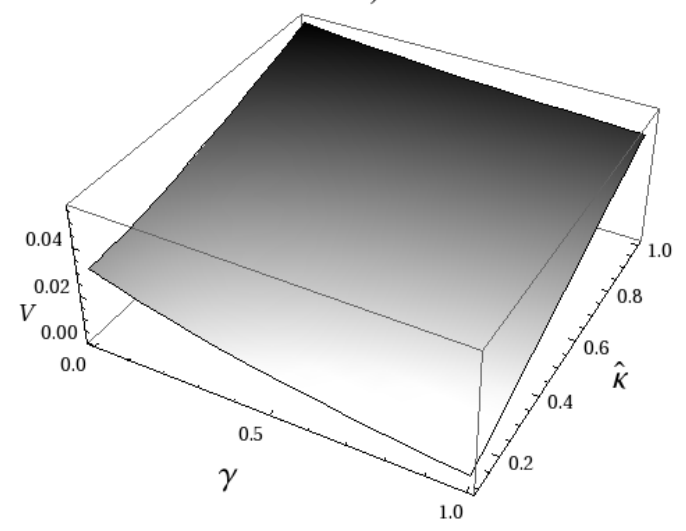

b.)

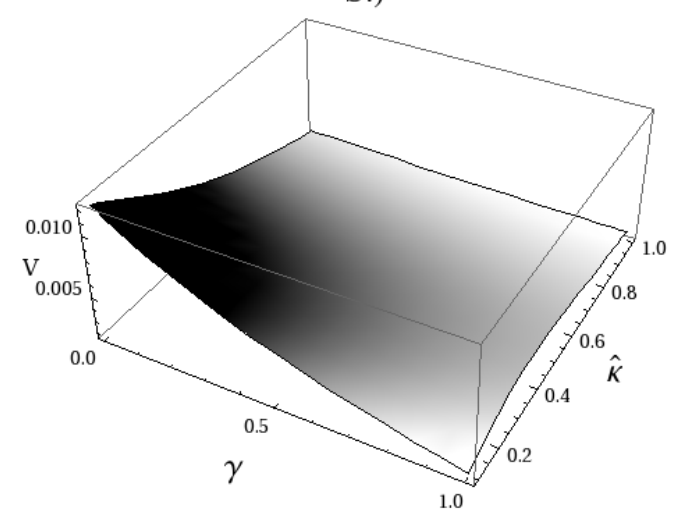

Fig. 3. Plots of $\tilde{V}_{H g}(\mathbf{s}, \mathbf{s})$ as a function $\gamma$ and of fixed $\hat{\kappa}$ taken at (a) $S=5.91$ and (b) $S=6.91$. Here, we have set the interface parameters at $\tilde{b}=5.91$ and $\tilde{a}=4.54$.

and therefore reducing $\zeta(\mathbf{s})$. Indeed, this effect increases as the local ionic concentration near the macro-ion increases.

We see that $\tilde{V}_{H g}(\mathbf{s}, \mathbf{s})$ increases with decreasing $\gamma$ (the ratio of dielectrics), as shown in Figure 3(a). This effect is explained by image charges of small ions. As an ion moves towards the macro-ion, it experiences repulsion due to its image charge. The size of these image charges depends on the value of $\gamma$. For instance, when $\gamma=1$ there are no image charges and at $\gamma=0$ the image charges are largest. As this is a positive contribution to $V_{H}(\mathbf{s}, \mathbf{s})$, this again reduces $\zeta(\mathbf{s})$.

Another qualitative feature we see in Figure 3(a) is that, as the local concentration of ions increases, the effect of image charge repulsion diminishes. This is because the other ions will try and weaken the image charge effect by adjusting themselves. The size of this adjustment depends on the local density of ions.

We can also look at the effect of distance on $\tilde{V}_{H g}(\mathbf{s}, \mathbf{s})$ by plotting $\tilde{V}_{H g}(\mathbf{s}, \mathbf{s})$ at $S=6.91$ in Figure $3(\mathrm{~b})$. When compared with Figure $3(\mathrm{a})$ we have that the dependence on $\hat{\kappa}$ has completely changed and the overall magnitude of $\tilde{V}_{H g}(\mathbf{s}, \mathbf{s})$ is reduced. This can be explained by both a reduction in the exclusion effects and image charge effects due the effects of more ions between an ion at $S=6.91$ and the macro-ion, than at $S=5.91$. More of these ions means that less of the local Debye atmosphere is lost and the image charge is screened more effectively.

Through the initial definition of $\zeta(\mathbf{s})$ (Eq. (5.5)) one is able to construct an equation to solve $\zeta(\mathbf{s})$. Then one is left with solving this system of equations numerically for both $\zeta(\mathbf{s})$ and $\phi_{0}(\mathbf{s})$. In Section 7 we will examine all these effects, in turn, for point ions.

\section{Dealing with finite ionic charge distributions}

\subsection{General formulation}

In this section we deal with the more realistic situation of extended charge distributions. We will suppose for simplicity, in the main text, that both species of ions have the same charge distribution $f_{+}\left(\mathbf{r}-\mathbf{r}_{j}^{+}, \boldsymbol{\omega}_{j}^{+}\right)=f_{-}(\mathbf{r}-$ $\left.\mathbf{r}_{j}^{+}, \boldsymbol{\omega}_{j}^{+}\right)=f\left(\mathbf{r}-\mathbf{r}_{j}^{+}, \boldsymbol{\omega}_{j}^{+}\right)$. Though, actually, the sizes and shapes of both electrolyte species can be quite different.

Using equations (2.2) and (2.14), it is possible to derive a field theory, albeit a non-local one, that takes account of these finite charge distributions

$$
Z_{\lambda}=\frac{1}{Z_{\mathrm{VAC}}} \int \mathcal{D} \phi(\mathbf{s}) \exp \left(-H_{\mathrm{ion}}[\phi(\mathbf{s})] / \Xi\right),
$$

where

$$
\begin{aligned}
& H_{\text {ion }}[\phi(\mathbf{s})]=\int \mathrm{d}^{3} s\left[\frac{1}{4} \tilde{\chi}(\mathbf{s})(\nabla \phi(\mathbf{s}))^{2}+i \tilde{\sigma}(\mathbf{s}) \phi(\mathbf{s})\right. \\
& \left.-\frac{\tilde{\kappa}^{2}}{2 \Lambda} \int \mathrm{d}^{3} s \int \frac{\mathrm{d}^{3} \omega}{8 \pi^{2}} \tilde{\Omega}_{I}(\mathbf{s}, \boldsymbol{\omega}) \cos \Phi(\mathbf{s}, \boldsymbol{\omega})\right]
\end{aligned}
$$

and

$$
\Phi(\mathbf{s}, \boldsymbol{\omega})=\int \mathrm{d}^{3} s^{\prime} \tilde{f}\left(\mathbf{s}-\mathbf{s}^{\prime}, \boldsymbol{\omega}\right) \phi\left(\mathbf{s}^{\prime}\right) .
$$

The rescaled form factor $\tilde{f}$ is normalized so that $\int \mathrm{d}^{3} s \tilde{f}(\mathbf{s}, \boldsymbol{\omega})=1$, i.e. $\tilde{f}(\mathbf{s}, \boldsymbol{\omega})=\mu^{3} f(\mathbf{r}, \boldsymbol{\omega})$. Furthermore, we have derived a more generalized field theory that does not assume that both species have the same shape. This can be found in Appendix D.

\subsection{Hartree equations}

From such a field theory it is possible to derive differointegral Hartree equations (see App. D).

$$
\begin{aligned}
& \tilde{\chi}(\mathbf{s}) \nabla_{\mathbf{s}}^{2} V_{H}\left(\mathbf{s}, \mathbf{s}^{\prime}\right)+\int \frac{\mathrm{d}^{3} \omega}{8 \pi^{2}} \int \mathrm{d}^{3} s_{1} \int \mathrm{d}^{3} s_{2} \\
& \times \tilde{f}\left(\mathbf{s}-\mathbf{s}_{1}, \boldsymbol{\omega}\right) \tilde{\Omega}_{I}\left(\mathbf{s}_{1}, \boldsymbol{\omega}\right) \tilde{\kappa}^{2} \zeta_{\text {ext }}\left(\mathbf{s}_{1}, \boldsymbol{\omega}\right) \cosh \Phi_{0}\left(\mathbf{s}_{1}, \boldsymbol{\omega}\right) \\
& \times \tilde{f}\left(\mathbf{s}_{1}-\mathbf{s}_{2}, \boldsymbol{\omega}\right) V_{H}\left(\mathbf{s}_{\mathbf{2}}, \mathbf{s}^{\prime}\right)=-2 \delta\left(\mathbf{s}^{\prime}-\mathbf{s}\right) \\
& \tilde{\chi}(\mathbf{r}) \nabla^{2} \phi_{0}(\mathbf{s})-\int \mathrm{d}^{3} s_{1} \int \frac{\mathrm{d}^{3} \omega}{8 \pi^{2}} \tilde{f}\left(\mathbf{s}-\mathbf{s}_{1}, \boldsymbol{\omega}\right) \tilde{\Omega}_{I}\left(\mathbf{s}_{1}, \boldsymbol{\omega}\right) \tilde{\kappa}^{2} \\
& \times \zeta_{\text {ext }}\left(\mathbf{s}_{1}, \boldsymbol{\omega}\right) \sinh \Phi_{0}\left(\mathbf{s}_{1}, \boldsymbol{\omega}\right)=-2 \tilde{\sigma}(\mathbf{s}),
\end{aligned}
$$


where $\Phi_{0}(\mathbf{s}, \boldsymbol{\omega})=\int \mathrm{d}^{3} s^{\prime} \tilde{f}\left(\mathbf{s}-\mathbf{s}^{\prime}, \boldsymbol{\omega}\right) \phi_{0}\left(\mathbf{s}^{\prime}\right)$ is the potential energy of a small ion, with orientation $\boldsymbol{\omega}$, in the average field produced by the macro-ion and the other small ions. Here again, there is a Boltzmann weight associated with the change in self-energy of an ion as it moves towards the macro-ion. This is

$$
\zeta_{\mathrm{ext}}(\mathbf{s}, \boldsymbol{\omega})=\exp \left(-\Xi\left(E_{H}(\mathbf{s}, \mathbf{s}, \boldsymbol{\omega})-E_{H}(\infty, \infty, \boldsymbol{\omega})\right) / 2\right),
$$

where $E_{H}(\mathbf{s}, \mathbf{s}, \boldsymbol{\omega})=\int \mathrm{d}^{3} s_{1} \int \mathrm{d}^{3} s_{2} \tilde{f}\left(\mathbf{s}-\mathbf{s}_{1}, \boldsymbol{\omega}\right) V_{H}\left(\mathbf{s}_{1}\right.$, $\left.\mathbf{s}_{2}\right) \tilde{f}\left(\mathbf{s}_{2}-\mathbf{s}, \boldsymbol{\omega}\right)$ is the ionic self-energy for an ion with orientation $\boldsymbol{\omega}$. In the Hartree approximation the local concentration of ions (with a given orientation $\boldsymbol{\omega}$ ), and the ionic charge density are given by $c(\mathbf{s}, \boldsymbol{\omega})=$ $\tilde{\kappa}^{2} \zeta_{\text {ext }}(\mathbf{s}, \boldsymbol{\omega}) \cosh \Phi_{0}(\mathbf{s}, \boldsymbol{\omega})$ and $\rho_{c}(\mathbf{s})=\int \mathrm{d}^{3} \omega \int \mathrm{d}^{3} r^{\prime} \tilde{f}(\mathbf{s}-$ $\left.\mathbf{s}^{\prime}, \boldsymbol{\omega}\right) \tilde{\kappa}^{2} \zeta_{\text {ext }}\left(\mathbf{s}^{\prime}, \boldsymbol{\omega}\right) \sinh \Phi_{0}\left(\mathbf{s}^{\prime}, \boldsymbol{\omega}\right)$, respectively.

Equations $(6.4,6.5)$ and $(6.6)$ constitute one of the main theoretical results of this paper. These equations may have greatest application when one considers extended molecules like cobalt hexamine or putracine, both important in the study of DNA. As was pointed out in [1], through simple arguments, taking account of finite-size effects for such molecules may be essential. Also, by appropriate choices of $\tilde{f}\left(\mathbf{s}-\mathbf{s}^{\prime}, \boldsymbol{\omega}\right)$, these equations may also be applied to the case of a macro-ion immersed in weakly interacting polar solvent, where the dipoles are considered explicitly. In this case, where $\Xi \rightarrow 0$, it should be possible to recover the results of [18].

However, as a preliminary investigation into the effect of the small ions having finite-size charge distributions, we shall consider only the simplest case of a spherically symmetric charge distribution, i.e. one that does not depend on $\boldsymbol{\omega}$. Then $\boldsymbol{\omega}$ may be simply integrated out and here on we will drop $\boldsymbol{\omega}$ from our results. Then, these equations considerably simplify (as shown in App. D). Now, by choosing an appropriate form for $\tilde{f}\left(\mathbf{s}^{\prime}-\mathbf{s}\right)$, considerable analytic progress can be made in solving them. We choose

$$
\tilde{f}\left(\mathbf{s}^{\prime}-\mathbf{s}\right)=\frac{1}{4 \pi} \frac{1}{r_{\text {ion }}^{2}} \frac{1}{\left|\mathbf{s}-\mathbf{s}^{\prime}\right|} \exp \left(-\left|\mathbf{s}-\mathbf{s}^{\prime}\right| / r_{\text {ion }}\right) \text {. }
$$

Indeed, the big advantage of (6.7) is that it is possible to derive an approximate partial solution to equation (6.4) as shown in Appendix D, by making the same WKB-like approximation. The result is, however, rather cumbersome so we refrain from quoting it the main text. But this again leaves us with a very much simplified integral equation on $\zeta_{\text {ext }}(\mathbf{s})$ which can be easily solved numerically in conjunction with the mean field. We denote the approximate terms in the self-energy, again, with a tilde on top, i.e. $\tilde{E}_{H}(\mathbf{s}, \mathbf{s})$.

Such a distribution does not accurately describe the charge distribution of a small ion, nevertheless equation (6.7) still describes important physics. For the small values of $r_{\text {ion }}$ that we consider, we find that $\Phi_{0}(\mathbf{s}) \approx \phi_{0}(\mathbf{s})$. So here, the charges may be considered point-like, so that $\phi_{0}(\mathbf{s})$ with $\Phi_{0}(\mathbf{s})$ does not matter and the actual form of the charge distribution is irrelevant. We find that the main effect of finite size comes within the self-energy $\tilde{E}_{H}(\mathbf{s}, \mathbf{s})$. The term $\tilde{E}_{H}(\mathbf{s}, \mathbf{s})$ may be divided into two pieces $\tilde{E}_{H g}(\mathbf{s}, \mathbf{s})$ (analogous to $\tilde{V}_{H g}(\mathbf{s}, \mathbf{s})$ for point charges) that describes effects due to macro-ion solvent interface and $\delta \tilde{E}_{I}(\mathbf{s}, \mathbf{s})$ (analogous to $\delta \tilde{V}_{I}(\mathbf{s}, \mathbf{s})$ for point charges) that describes local changes in the electrostatic self-energy of an ion due to the varying concentration of ions. Now, any charge smearing due to a finite charge distribution on ions causes a reduction in $\tilde{E}_{H g}(\mathbf{s}, \mathbf{s})$, due to a reduction in image charge repulsion, which is captured in equation (6.7). Though, we find that the term most sensitive to finite-size effects is $\delta E_{I}(\mathbf{s}, \mathbf{s})$. Here they do make quite a difference. This term is essentially a localized solvation energy [47]. Indeed equation (6.7) may work reasonably, provided that the parameter $r_{\text {ion }}$ is correctly related to ionic size.

\section{Results for the uniformly charged macro-ion}

\subsection{Point charges}

\subsubsection{The equations}

In these calculations, we consider only univalent ions and calculate both the correlation parameter and GouyChapman lengths for a surface charge density of $\sigma=$ $16.8 \mu \mathrm{C} / \mathrm{cm}^{2}$, which is the value for DNA, for which we find values of $\Xi \approx 20$ [48] and $\mu \approx 2.2 \AA$. For different charge densities, comparable with DNA, it is more convenient to keep these values fixed, instead of recalculating them for each charge density.

In what follows, we will suppose a uniform charge distribution on the macro-ion so that $\tilde{\sigma}(\mathbf{s})=\tilde{\sigma}(S)=$ $-\sigma_{f} \delta(S-\tilde{a})[49]$; here we take $0 \leq \sigma_{f} \leq 1$. The quantity $\sigma_{f}$ represents a fraction of the DNA charge density. So, we may consider different fixed surface charge densities and keep $\Xi$ and $\mu$ fixed. We take the radius of the core region to be $\tilde{a}=4.54$ Gouy-Chapman lengths $(\approx 10 \AA)$, roughly that of a DNA molecule. We allow the closest approach distance from the central axes of the macro-ion, for the small ions, to be $\tilde{b}=5.91$ (in $\mu$, the Guoy-Chapman length). Therefore, we assign each ion an effective hardcore radius of $1.37 \mu(\approx 3 \AA)$. This roughly corresponds to a small ion (for instance sodium) surrounded by a tightly bound first hydration shell of water. The key assumption being: the electrostatic field is not strong enough to partially remove this hydration shell from a significant fraction of ions close to the macro-ion.

Before working with the equations numerically, it is convenient to recast them in an integral equation form. We may define a quantity called the excess charge density, $\rho_{\text {ex }}(S)$ so that

$$
\begin{aligned}
\tilde{\chi}(S) & {\left[\frac{\mathrm{d}^{2} \phi_{0}(S)}{\mathrm{d} S^{2}}+\frac{1}{S} \frac{\mathrm{d} \phi_{0}(S)}{\mathrm{d} S}\right]-\tilde{\Omega}_{I}(S) \tilde{\kappa}^{2} \phi_{0}(S)=} \\
& -2 \tilde{\sigma}(S)+\tilde{\Omega}_{I}(S) \rho_{\mathrm{ex}}(S) .
\end{aligned}
$$


It is then possible to show that $\rho_{\mathrm{ex}}(S)$ must satisfy the following integral equation:

$$
\begin{aligned}
\rho_{\mathrm{ex}}(S)= & \kappa^{2} \zeta(S) \int_{\tilde{b}}^{\infty} S^{\prime} \mathrm{d} S^{\prime} \bar{G}\left(S, S^{\prime}\right) \\
& \times\left(\frac{2 \sigma_{f} \tilde{a}}{\tilde{b}} \delta\left(S^{\prime}-\tilde{b}\right)+\rho_{\mathrm{ex}}\left(S^{\prime}\right)\right) \\
& -\kappa^{2} \zeta(S) \sinh \int_{\tilde{b}}^{\infty} S^{\prime} \mathrm{d} S^{\prime} \bar{G}\left(S, S^{\prime}\right) \\
& \times\left(\frac{2 \sigma_{f} \tilde{a}}{\tilde{b}} \delta\left(S^{\prime}-\tilde{b}\right)+\rho_{\mathrm{ex}}\left(S^{\prime}\right)\right),
\end{aligned}
$$

where

$$
\begin{aligned}
& \bar{G}\left(S, S^{\prime}\right)=\hat{G}\left(S, S^{\prime}\right) \theta\left(S-S^{\prime}\right)+\hat{G}\left(S^{\prime}, S\right) \theta\left(S^{\prime}-S\right), \quad(7.3) \\
& \hat{G}\left(S, S^{\prime}\right)=\frac{K_{0}(\tilde{\kappa} S)}{K_{1}(\tilde{\kappa} \tilde{b})}\left(K_{0}\left(\tilde{\kappa} S^{\prime}\right) I_{1}(\tilde{\kappa} \tilde{b})+I_{0}\left(\tilde{\kappa} S^{\prime}\right) K_{1}(\tilde{\kappa} \tilde{b})\right),
\end{aligned}
$$

and $\mu \tilde{b}=b$. The reduced electrostatic potential is determined through

$$
\phi_{0}(S)=\int_{\tilde{b}}^{\infty} S^{\prime} \mathrm{d} S^{\prime} \bar{G}\left(S, S^{\prime}\right)\left(2 \sigma\left(S^{\prime}\right)+\rho_{\mathrm{ex}}\left(S^{\prime}\right)\right) .
$$

Using our approximation, our equation on $\zeta(S)$ reads as

$$
\zeta(S)=\exp \left(-\Xi / 2\left(\tilde{V}_{H g}(S, S)+\delta V_{I}(S, S)\right)\right),
$$

where $\delta V_{I}(S, S)$ and $\tilde{V}_{H g}(S, S)$ are given by equations (5.11) and (5.13). Now $\hat{\kappa}(\mathbf{s}, q)$ depends spatially only on $S$ through $\zeta(S)$ and $\phi_{0}(S)$.

Now, to examine the various effects, we shall consider three scenarios for calculating $\zeta(S)$ for point charges, based on the following assumptions:

1) We solve equation (7.2) with $\zeta(S)=1$; this corresponds to the PB equation. No correlation, image charge or exclusion effects are considered.

2) In the calculation of $\zeta(S)$ we allow small ions to freely penetrate into the macro-ion. Also, we assume a uniform dielectric constant of $\varepsilon_{w} \simeq 80$ throughout. Therefore, we set $\tilde{V}_{H g}(S, S)=0$ in equation (7.6), determining $\zeta(S)$, in our calculations.

3) Exclude ions from the macro-ion core but keep the delectric constant throughout the space at $\varepsilon_{w} \approx 80$, then $\gamma=1$ in equation (5.14).

4) The full theory. Assume that we have a dielectric interface for which $\varepsilon_{w} \gg \varepsilon_{c}$ with $\varepsilon_{w} \approx 80$ we, then, have $\gamma=0$ in equation (5.14).

In numerically solving these equations we use an iterative technique. We start with initial guesses (trial functions) for both $\zeta(S)$ and $\rho_{\mathrm{ex}}(S)$, which are $\zeta_{0}(S)$ and $\rho_{0}(S)$. These are then fed into the R.H.S. of both equations (7.2) and (7.6), and new values $\zeta_{1}(S)$ and $\rho_{1}(S)$ are obtained from the L.H.S. of both equations. Due to the strong non-linear nature of these equations, we then use a particular algorithm to improve the convergence rate [50]. Instead of $\rho_{1}(S)$, we use $\bar{\rho}_{1}(S)=0.5 \rho_{1}(S)+0.5 \rho_{0}(S)$. Both $\zeta_{1}(S)$ and $\bar{\rho}_{1}(S)$ are then inserted back into the R.H.S. of equations (7.2) and (7.6), so $\zeta_{2}(S)$ and $\bar{\rho}_{2}(S)$ are calculated. One then iterates the process. But, crucially at the $n$-th step, one uses the values $\zeta_{n-1}(S)$ and $\bar{\rho}_{n-1}(S)=0.5 \bar{\rho}_{n-2}(S)+0.5 \rho_{n-1}(S)$ to calculate both $\zeta_{n}(S)$ and $\rho_{n}(S)$. We iterate as many times as is required to obtain accurate values of $\zeta(S)$ and $\rho_{\mathrm{ex}}(S)$. This is achieved when $\zeta_{n}(S) \simeq \zeta_{n-1}(S)$ and $\rho_{n}(S) \simeq \rho_{n-1}(S)$, which we ensure to a high accuracy.

\subsubsection{Numerical results}

Unfortunately, we were not able to obtain solutions in all three situations for $\sigma_{f}=1$, the full DNA charge. This simply may be due to the fact that our approximation that we use in solving Hartree equations no longer works at this fixed surface charge density. The WKB-like approximation seems to break down at the lowest values of $\sigma_{f}$ when no boundary effects are included (scenario 2), this seems to make sense as the variation in $c(S)$ for this scenario becomes very large with $S$ at values of $\sigma_{f}$ just below this. However, we are able to obtain solutions at $\sigma_{f}=0.5, \tilde{\kappa}=0.48$, for all four scenarios, which are shown in Figure 4.

We notice in Figure 4a that the magnitudes of the potentials calculated with correlation effects all lie below those calculated with the PB equation. Those calculated in scenarios 3) and 4) lie very close to the PB result (scenario 1)). For scenario 2) (no interface assumed in calculating $\zeta(S)$ ) the calculated potential lies much further away from the $\mathrm{PB}$ result. In Figure $4 \mathrm{~b}$ we calculate the local concentration of small ions. We see that when there is no interface the concentration is much larger than the $\mathrm{PB}$ equation and simply monotonically increases, whereas, for scenarios 3) and 4) the local concentration is roughly the same as that calculated for the PB equation. However, there is one different qualitative feature that both these solutions exhibit; the concentration starts to fall as one moves closer to the surface of the molecule. This is explained when we come to examine the correlation parameter $\zeta(S)$. First we see that, at large enough distances away from the macro-ion, the calculated values of $\zeta(S)$ for scenarios 2), 3) and 4) all start to increase with decreasing $S$. The amount of correlations increases due to an increase in the local density of ions; there are more ions to adjust to the presence of a single ion at a particular instance. But also, we see that for scenarios 3) and 4), $\zeta(S)$ exhibits a distinct dip as we come close to the surface of the macro-ion. In scenario 3 ) this dip is due only to the exclusion of ions from the macro ion, the physics of which was discussed at the end of Section 5. Essentially as an ion comes very close to the surface, less ions contribute to the screening out of its electric field, so its electrostatic self-energy starts to increase, which reduces $\zeta(S)$. When image charge effects are considered, in scenario 4$)$, this dip becomes more pronounced. This is because the electrostatic self-energy of ions is increased by image charge repulsion (see Sect. 5).

In Figure 5 we examine how the results change when we decrease $\sigma_{f}$, the relative surface charge density, by showing results calculated for $\sigma_{f}=0.25, \tilde{\kappa}=0.48$. For the potentials calculated in scenarios 3 ) and 4), the 
a.)

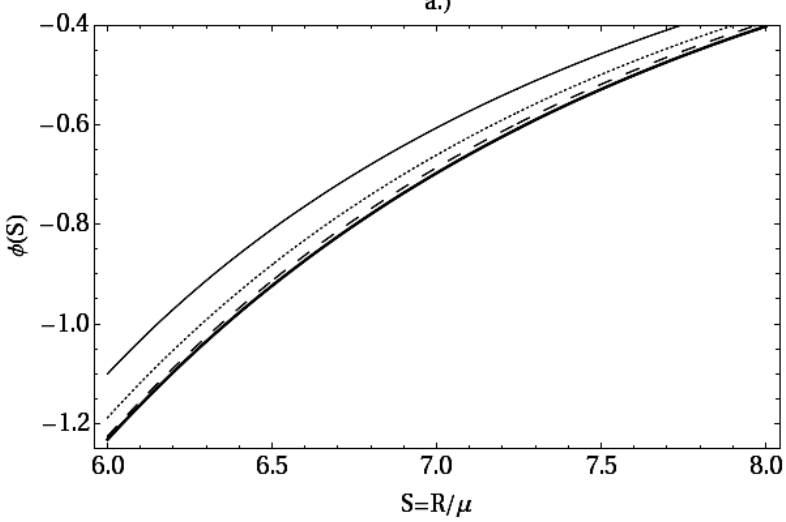

b.)

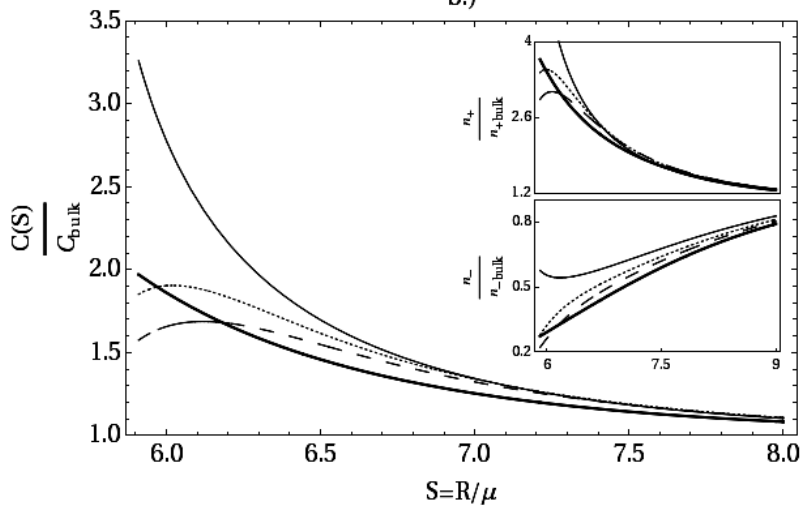

c.)

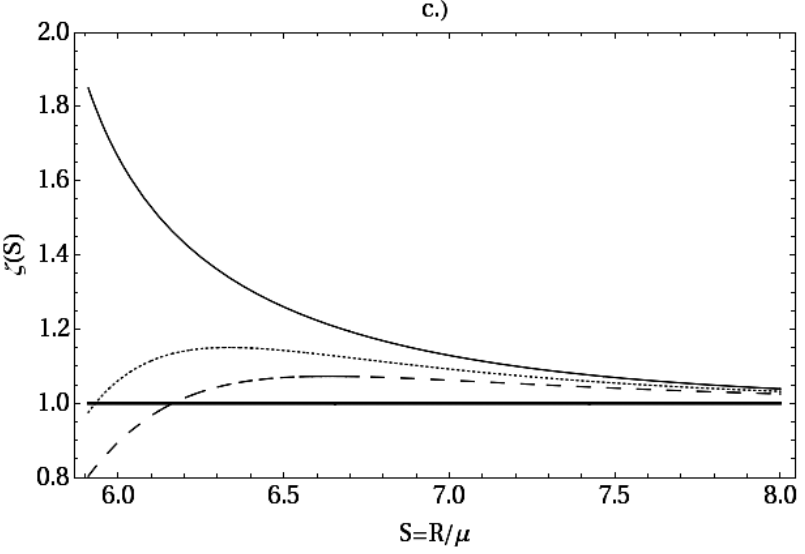

Fig. 4. Results for point charges at 50\% DNA surface charge density with $\tilde{\kappa}=0.48$. a) For the electrostatic potential. b) The local relative concentration of ions to the bulk $c(S) / c_{\text {bulk }}$. Here the insets show the relative concentrations of positive $n_{+}(S) / n_{+ \text {bulk }}$ and negative ions $n_{-}(S) / n_{- \text {bulk }}$ c) For the correlation parameter $\zeta(S)$. In all three figures, the thick solid line corresponds to the solution of the standard $\mathrm{PB}$ equation. The thin solid line corresponds to calculations in scenario 2). The dotted line is the situation where the dielectric is uniform, but ions are not allowed to penetrate the macro-ion. The dashed line is when the dielectric core has a considerably smaller dielectric constant compared to the surrounding solvent.

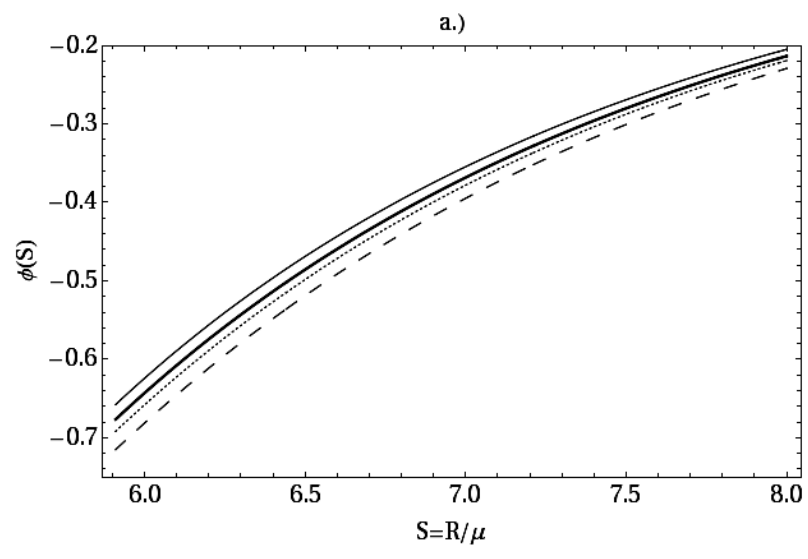

b.)

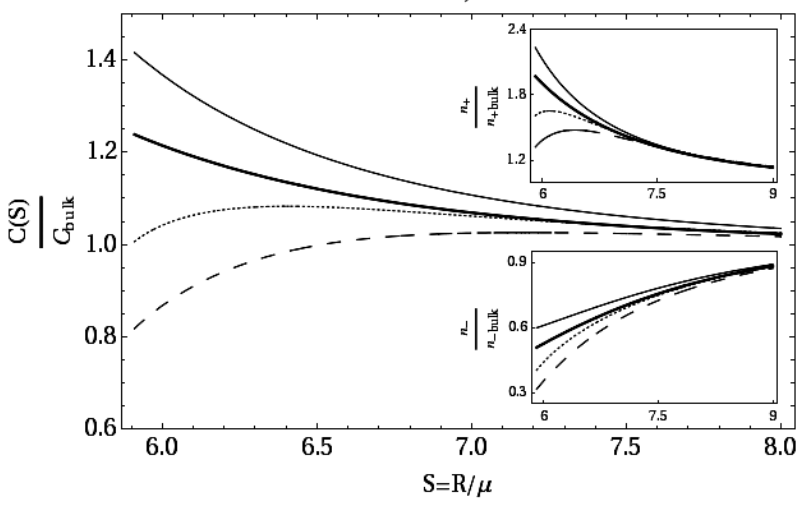

c.)

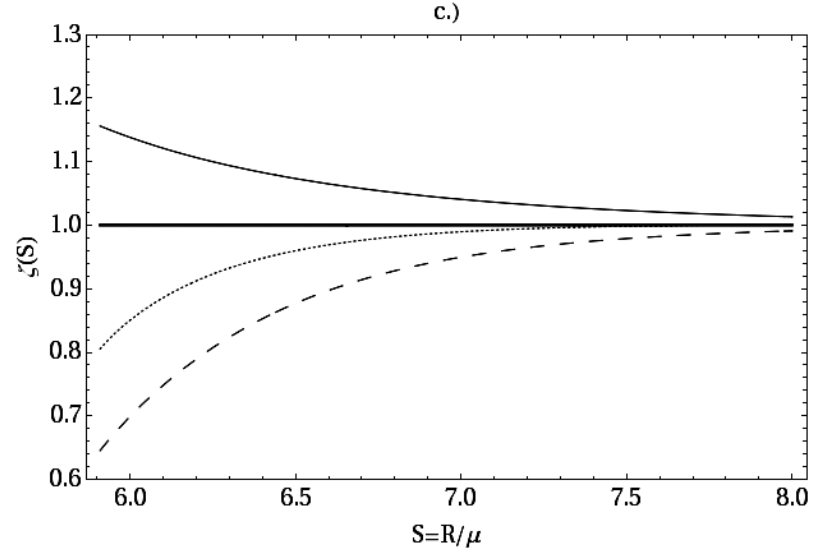

Fig. 5. Results for point charges at $25 \%$ DNA surface charge density with $\tilde{\kappa}=0.48$. a) For the electrostatic potential. b) The local relative concentration of ions to the bulk $c(S) / c_{\text {bulk }}$. Here the insets show the relative concentrations of positive $n_{+}(S) / n_{+ \text {bulk }}$ and negative ions $n_{-}(S) / n_{- \text {bulk }}$ c) For the correlation parameter $\zeta(S)$. In all, the thick solid line corresponds to the solution of the standard PB equation. The thin solid line corresponds to the solution where no interface is assumed in calculating $\zeta(S)$ : the dielectric is uniform and ions are allowed to penetrate the macro-ion. The dotted lines correspond to the situation where the dielectric is uniform, but ions are not allowed to penetrate the macro-ion. The dashed lines correspond to the situation where the dielectric core has a considerably smaller dielectric constant compared to the surrounding solvent. 


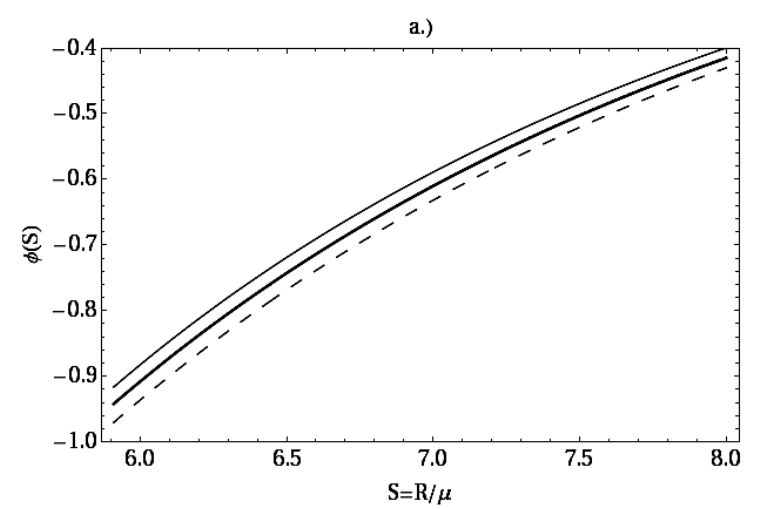

b.)

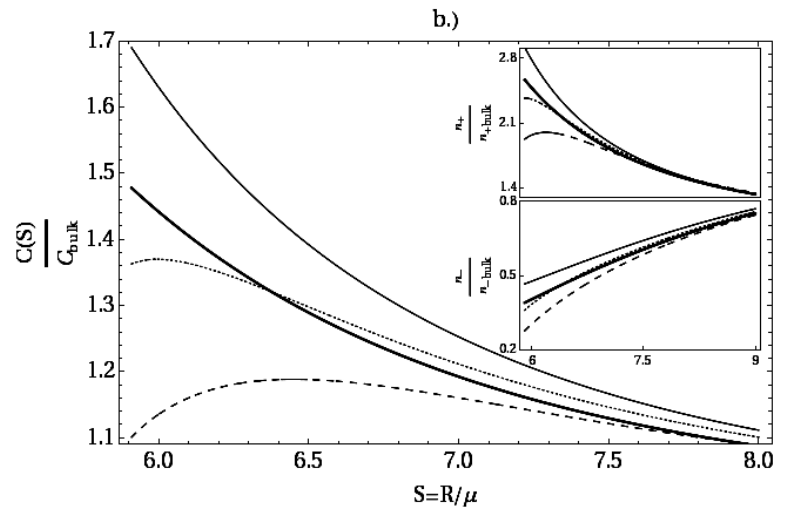

c.)

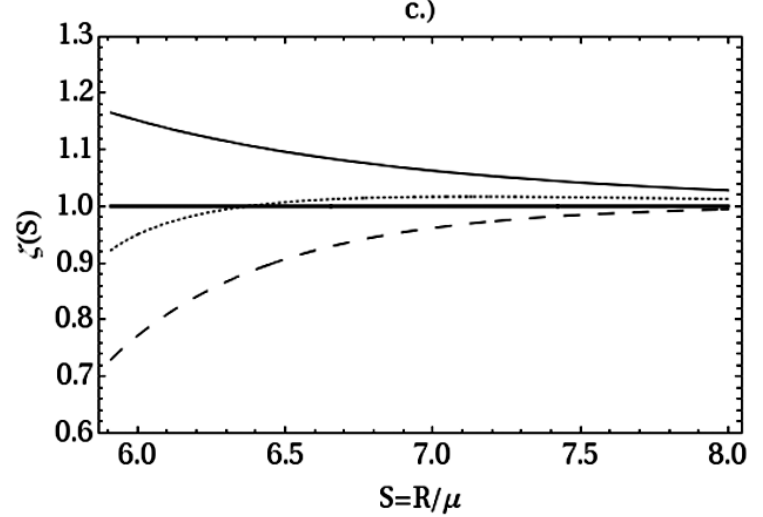

Fig. 6. Results for point charges at $25 \%$ DNA surface charge density with $\tilde{\kappa}=0.32$. a) For the electrostatic potential. b) The local relative concentration of ions to the bulk $c(S) / c_{\text {bulk }}$. Here the insets show the relative concentrations of positive $n_{+}(S) / n_{+ \text {bulk }}$ and negative ions $n_{-}(S) / n_{- \text {bulk }}$. c) For the correlation parameter $\zeta(S)$. Shown in all is the solution of the standard PB equation (thick solid line), the solution with correlations in $\zeta(S)$ but no interface (thin solid line), the situation where the dielectric is uniform but ions are excluded from the marco-ion (dots), and the situation where the dielectric core has a considerably smaller dielectric constant compared to the surrounding solvent (dashes). Note that in a) the solution for scenario 3) is indistinguishable from the PB result.

magnitude of the electrostatic potential is larger than the $\mathrm{PB}$ result (Fig. 5a). We see that in Figure 5b the calculated local concentration of small ions without an interface (scenario 2)) is still larger than the PB result, but not so dramatically as what was calculated at $\sigma_{f}=0.5$. However now, the local concentrations calculated in scenarios 3) and 4) are much lower than what has been calculated for the PB equation. The reduction in concentration seen for these two results, at small $S$ as we move close to the macro-ion, is more pronounced. When we calculate the correlation parameter $\zeta(S)$, we see that, now, for scenarios 3) and 4) $\zeta(S)<1$ for most values of $S$. This reduction in $\zeta(S)$, from the results calculated at $\sigma_{f}=0.5$, comes from a reduction in correlation effects, which increase $\zeta(S)$. This reduction is simply because the local concentration close to the macro-ion is much reduced from that of $\sigma_{f}=0.5$; there is less electrostatic attraction between the bound surface charges of the macro-ion and the small positive ions in solution. Effects due to the macro-ion solvent boundary dominate, simply making it unprofitable for ions, in terms of their electrostatic self energy, to come close the macro-ion; they lose part of their Debye atmosphere and see image charge reflections (in scenario 4)). Indeed, the correlation parameter $\zeta(S)$ is smallest when a small dielectric constant is considered for the macro-ion core.

In Figure 6 we keep $\sigma_{f}$ fixed, but change $\tilde{\kappa}$ and consider $\sigma_{f}=0.25$ and $\tilde{\kappa}=0.32$. By comparing this with Figure 5, it seems that, as one lowers the salt concentrations, the effect of excluding ions is reduced and the effect of introducing low dielectric constant in macro-ion core is increased. We also see that the correlation parameter $\zeta(S)$ is pushed up slightly for all the results. This is explained by an increase in the local relative concentration of small ions which, in turn, increases the correlation effects.

Lastly for point charges, in Figure 7 we calculate the charge density of small ions. When there are no interface effects in $\zeta(S)$, scenario 2), the charge density is highest. This is indeed what causes the lowest-magnitude electrostatic potential of the four scenarios considered. At $\sigma_{f}=0.5$ both the charge densities calculated for scenarios 3) and 4) lie close to the PB result. Furthermore, at $\sigma_{f}=0.25$ the results for scenario 3) (ionic exclusion, no image charges) is almost identical to that of the PB equation. However, at this fixed surface charge density, the result for scenario 4) (image charges included) do show some significant deviation away from the PB result at low values of $S$; the charge density of small ions is lower for the former result. For completeness, in these figures, in the insets we show $\rho_{\text {ex }}(S)$. Clearly one sees that, in all cases at $\sigma_{f}=0.5$, the fully linearized PB equation $\left(\rho_{\mathrm{ex}}(S)=0\right)$ does not work. At $\sigma_{f}=0.25$, linearization works reasonably well, except for scenario 2). The function $\rho_{\mathrm{ex}}(S)$ is useful when we consider the theory of interaction between macro-molecules. Here, it can be used to calculate a renormalized charge in the DLVO approximation, used for such a theory, as we shall do later.

\subsubsection{First-order corrections}

We may compute the first correction to the WKB-like approximation, employed for point charges in the numerical calculations of this paper, from the exact solution of the Hartree equations. The correction is given by

$$
\begin{aligned}
& \Delta \tilde{V}_{H}(\mathbf{s}, \mathbf{s})=-\frac{\tilde{\kappa}^{2}}{2} \int \mathrm{d}^{3} \mathbf{s}^{\prime \prime} \tilde{V}_{H}\left(\mathbf{s}, \mathbf{s}^{\prime \prime}\right) \Omega_{I}\left(\mathbf{s}^{\prime \prime}\right) \\
& \times\left(\lambda\left(S^{\prime \prime}\right) \cosh \phi_{0}\left(S^{\prime \prime}\right)-\lambda(S) \cosh \phi_{0}(S)\right) \tilde{V}_{H}\left(\mathbf{s}^{\prime \prime}, \mathbf{s}\right) .
\end{aligned}
$$


a.)

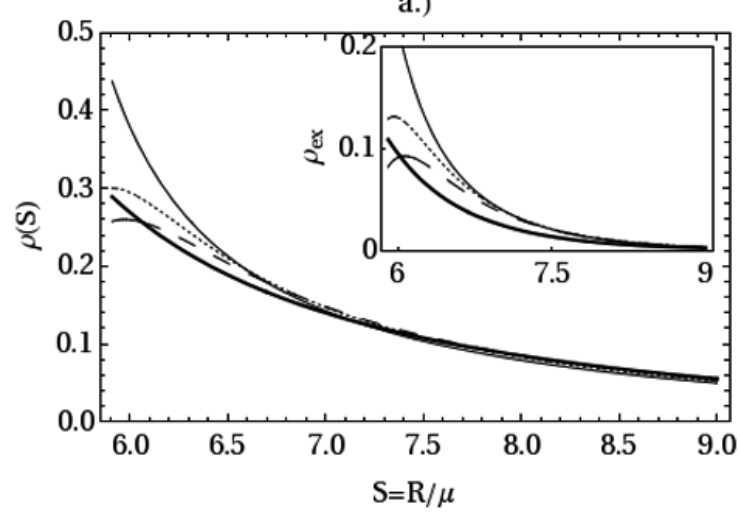

b.)

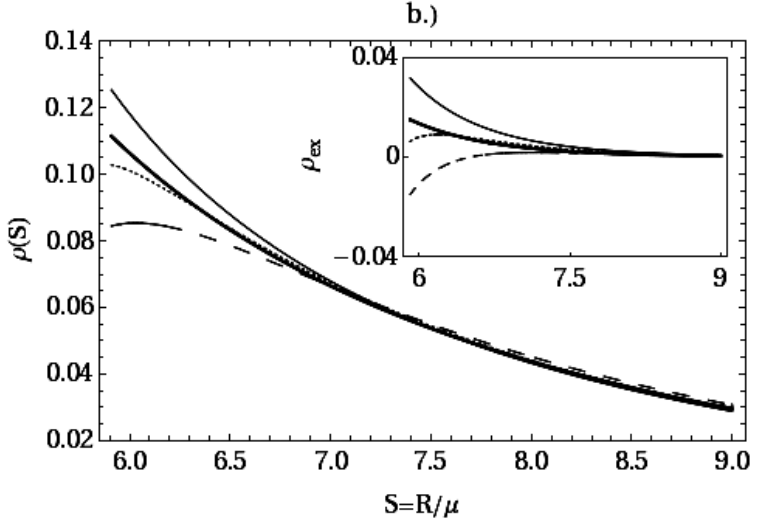

Fig. 7. Results for the charge density of small ions $\rho(S)$. The insets show the excess charge density, $\rho_{\mathrm{ex}}(S)$ defined in equation (7.2). Here $\tilde{\kappa}=0.32$. a) At $50 \%$ DNA charge density. b) At $25 \%$ DNA charge density. The thick solid line corresponds to the solution of the standard PB equation. The thin solid line corresponds to the solution calculated in scenario 2). The dotted line corresponds to the situation where the dielectric is uniform, but ions are not allowed to penetrate the macro-ion. The dashed lines correspond to the situation where the dielectric core has a considerably smaller dielectric constant compared to the surrounding solvent.

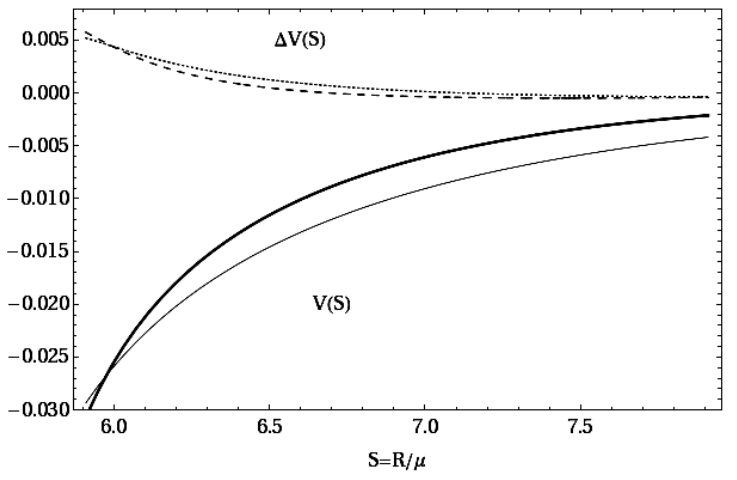

Fig. 8. Figure showing both the correction $\Delta V(S)=$ $\Delta \tilde{V}_{H}(\mathbf{s}, \mathbf{s}) / 2$ and $V(S)=\left(\tilde{V}_{H}(\mathbf{s}, \mathbf{s})-\tilde{V}_{H}(\infty, \infty)\right) / 2$ for $\sigma_{f}=0.5$ for the case where there in no interface (scenario 2)). The quantity $V(S)$ is plotted with a thick solid line (for $\tilde{\kappa}=0.48$ ) and a thin solid line (for $\tilde{\kappa}=0.32$ ), whereas the correction is plotted using a short-dashed line (for $\tilde{\kappa}=0.48$ ) and a dotted line (for $\tilde{\kappa}=0.32)$.

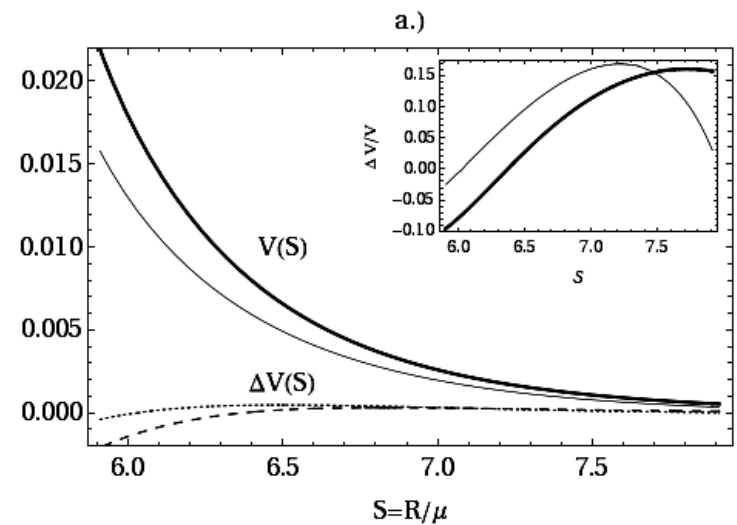

b.)

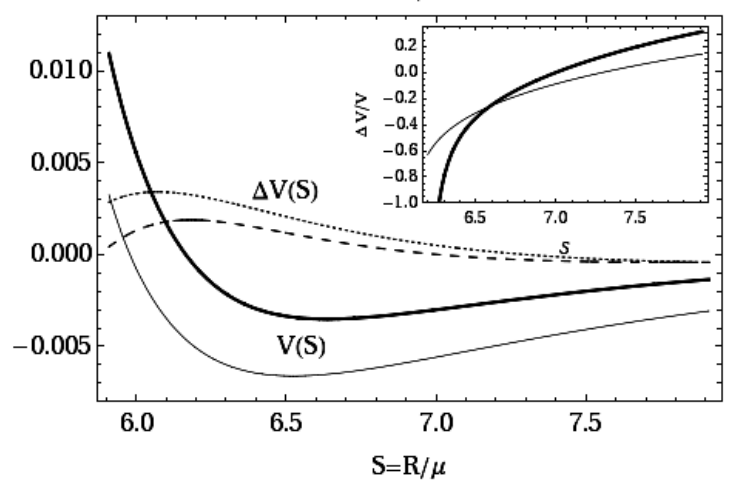

c.)

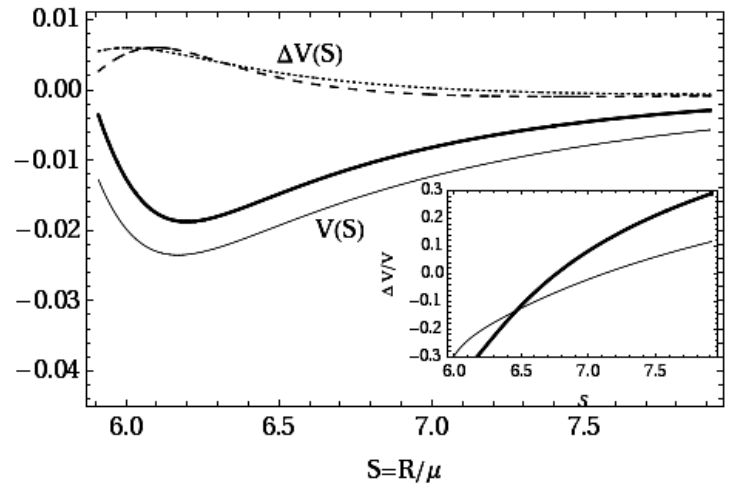

Fig. 9. Figures showing both the correction $\Delta V(S)=$ $\Delta \tilde{V}_{H}(\mathbf{s}, \mathbf{s}) / 2$ and $V(S)=\left(\tilde{V}_{H}(\mathbf{s}, \mathbf{s})-\tilde{V}_{H}(\infty, \infty)\right) / 2$ for a) $\sigma_{p}=$ 0.25 , b) $\sigma_{p}=0.5$ and c) $\sigma_{p}=0.75$. The insets show the ratio of the these two quantities. Main figures: the quantity $V(S)$ is plotted with a thick solid line (for $\tilde{\kappa}=0.48$ ) and a thin solid line (for $\tilde{\kappa}=0.32$ ), whereas the correction is plotted using a short dashed line (for $\tilde{\kappa}=0.48$ ) and a dotted line (for $\tilde{\kappa}=0.32$ ). Insets: thick solid line for $\tilde{\kappa}=0.48$ and thin solid line for $\tilde{\kappa}=0.32$.

In Appendix $\mathrm{C}$ we recast equation (7.7) in a more explicit, but cumbersome way, which can readily be used for numerical calculation.

We calculate this correction in two of the scenarios previously discussed: scenario 2), where there is no interface, and for scenario 4), where we have the full interface.

In Figure 8 we show the correction $\Delta \tilde{V}_{H}(\mathbf{s}, \mathbf{s})$ in comparison to $\tilde{V}_{H}(\mathbf{s}, \mathbf{s})-\tilde{V}_{H}(\infty, \infty)$ for scenario 2$)$. As 
one sees the correction is relatively small in relation to $\tilde{V}_{H}(\mathbf{s}, \mathbf{s})-\tilde{V}_{H}(\infty, \infty)$. This is also true when smaller values of $\sigma_{f}$ are considered.

The size of the correction $\Delta \tilde{V}_{H}(\mathbf{s}, \mathbf{s})$ in comparison to $\tilde{V}_{H}(\mathbf{s}, \mathbf{s})-\tilde{V}_{H}(\infty, \infty)$, for scenario 4$)$, is shown in Figure 9 for $\sigma_{f}=0.75,0.5$ and 0.25 . At $\sigma_{f}=0.25$, the correction is small in relation to $\tilde{V}_{H}(\mathbf{s}, \mathbf{s})-\tilde{V}_{H}(\infty, \infty)$, though significant. However, in the region of the parameters explored, we see that the WKB-like approximation works less well in situations where repulsive effects -image charges and exclusion of ions - and correlation effects are of similar size. This is the case when $\sigma_{p}=0.5$. Here, there are small regions in $S$ where the correction is of similar size or even larger. This is due to the fact that $\tilde{V}_{H}(\mathbf{s}, \mathbf{s})-\tilde{V}_{H}(\infty, \infty)$ changes sign, moving from a region where image charges and ionic exclusion effects dominate to a region where correlation effects dominate. For $\sigma_{f}=0.75$, the correction becomes quite large at the largest and smallest values of $S$ for which it is calculated, but still remains smaller than $\tilde{V}_{H}(\mathbf{s}, \mathbf{s})-\tilde{V}_{H}(\infty, \infty)$. In most cases, we see that the WKBlike approximation does not work so well for $\tilde{\kappa}=0.48$ than it does for $\tilde{\kappa}=0.32$.

Nevertheless, the overall conclusion here is, for the most part, that the WKB-like approximation does not perform too badly, although the correction can be significant. Certainly, it works well enough to give insight into the qualitative physics. Of course, better quantitative accuracy can be sought for by either incorporating the correction $\Delta \tilde{V}_{H}(\mathbf{s}, \mathbf{s})$ into the calculation of the electrostatic potential or working with the exact Hartree equations, in an integral equation form, from which the WKB-like approximation could form a suitable starting point (see App. C for point charges).

\subsection{Extended charges}

\subsubsection{Equations for the uniformly charged cylinder}

Again we consider a uniform charge distribution on the macro ion, again of the same form. Here, one can introduce radial smearing of the fixed charge groups. But for simplicity, and purposes of comparison, we keep the same charge distribution as before.

The ionic potential energy $\Phi_{0}(S)$ is determined through

$$
\Phi_{0}(S)=\int_{0}^{\infty} S^{\prime} \mathrm{d} S^{\prime} \overline{\mathcal{G}}\left(S, S^{\prime}\right) \eta\left(S^{\prime}\right)
$$

An explicit expression for the Green's function $\overline{\mathcal{G}}\left(S, S^{\prime}\right)$ is cumbersome and is left to Appendix D. The quantity $\eta\left(S^{\prime}\right)$ may be determined self-consistently through

$$
\begin{aligned}
& \eta(S)=\kappa^{2} \int_{0}^{\infty} \mathrm{d} S^{\prime} S^{\prime} \overline{\mathcal{G}}\left(S, S^{\prime}\right) \eta\left(S^{\prime}\right) \\
& -\kappa^{2} \sinh \int_{0}^{\infty} \mathrm{d} S^{\prime} \mathcal{S}^{\prime} \overline{\mathcal{G}}\left(S, S^{\prime}\right) \eta\left(S^{\prime}\right), \quad \text { for } S>\tilde{b}, \\
& \eta(S)=\left[-\frac{\mathrm{d}^{2}}{\mathrm{~d} S^{2}}-\frac{1}{S} \frac{\mathrm{d}}{\mathrm{d} S}+\frac{1}{r_{\text {ion }}^{2}}\right] 2 \tilde{\sigma}(S), \quad \text { for } S<\tilde{b} .
\end{aligned}
$$
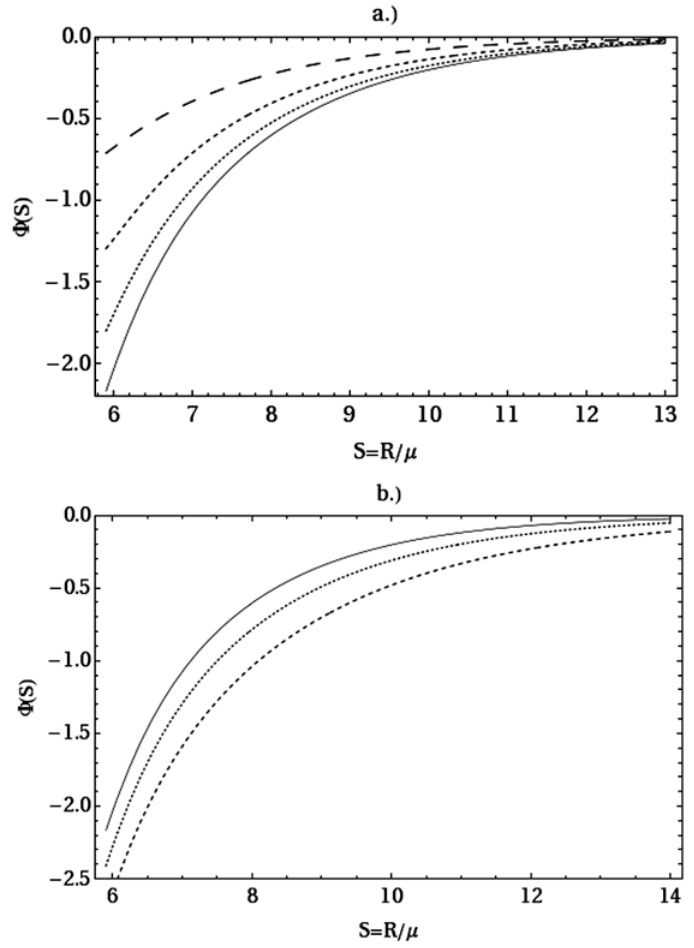

Fig. 10. Figure showing the ionic potential energy, $\Phi(S)$ for $r_{\text {ion }}=0.185$. a) For $\tilde{\kappa}=0.48$, with $100 \%$ (solid line), $75 \%$ (dotted line), $50 \%$ (short dashes) and 25\% (long dashes) of DNA charge. b) At $100 \%$ DNA surface charge density at $\tilde{\kappa}=$ 0.48 (solid line), $\tilde{\kappa}=0.4$ (dotted line) and $\tilde{\kappa}=0.32$ (short dashes).

Now, $\zeta(S)$ (defined as $\zeta_{\text {ext }}(S)$ in previous section) is selfconsistently determined through

$$
\zeta(S)=\exp \left(-\Xi / 2\left(\tilde{E}_{H g}(S, S)+\delta \tilde{E}_{I}(S, S)\right)\right)
$$

where expressions for $\tilde{E}_{H g}(S, S)$ and $\delta E_{I}(S, S)$ (in terms of $\zeta(S))$ are given in Appendix D.

In the limit $r_{\text {ion }} \rightarrow 0$, equations $(7.8,7.9)$ and $(7.11)$ reproduce equations $(7.5,7.2)$ and $(7.6)$. These equations are solved in an iterative manner similar to those of the point charges. The difference is that instead of using $\rho_{\mathrm{ex}}(S)$, $\eta(S)$ is used along with $\zeta(S)$ in the iterative procedure.

For the size of our charge distributions, we consider $r_{\text {ion }}=0.185$ and $r_{\text {ion }}=0.25$ (sizes given in GouyChapman lengths) [51]. Not very large values of $r_{\text {ion }}$ are chosen for the following reason. As the charge distribution of the small ions becomes very smeared out, some quite non-trivial behaviour is expected. There is a possibility for such sizes, that there will be oscillatory behavior in the solutions of equations (7.9) and (7.11). This is because, at sufficiently large values of $r_{\text {ion }}$, two of the three decay lengths present in both $\overline{\mathcal{G}}\left(S, S^{\prime}\right)$ and $E(S, S)$ may become complex (see App. D). This change in the decay lengths has not been taken into account in numerical calculations [52].

\subsubsection{Numerical results}

We obtain solutions both for different values of $\sigma_{f}$ and $\tilde{\kappa}$. In Figure 10 we present results for the ionic potential 

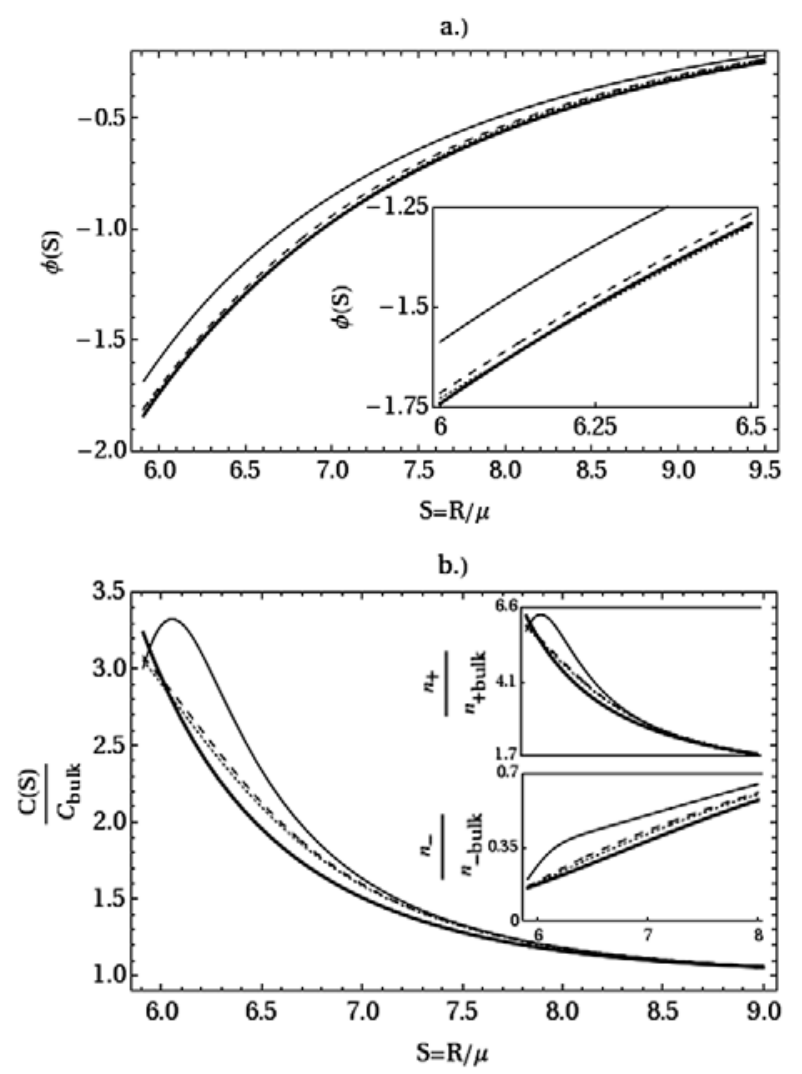

c.)

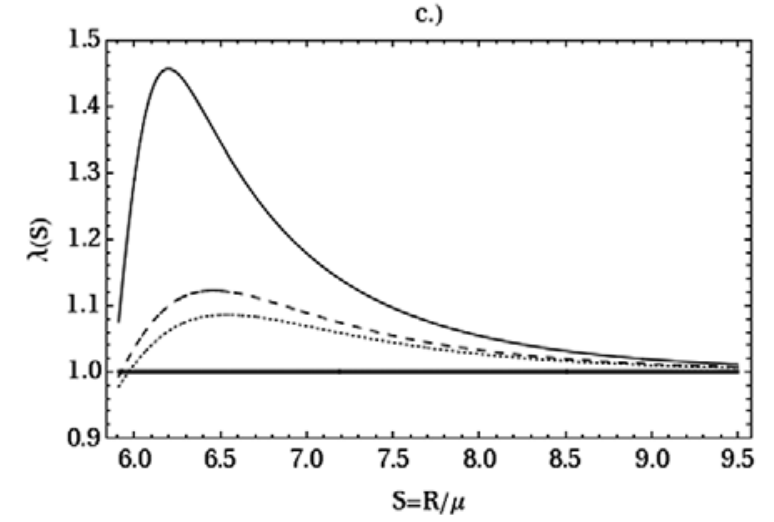

Fig. 11. Results of numerical calculations at $75 \%$ DNA charge density at $\tilde{\kappa}=0.48$. a) For the electrostatic potential. b) For local concentration of small ions $c(S) / c_{\text {bulk }}$ to the bulk. Here the insets show the relative concentrations of positive $n_{+}(S) / n_{+ \text {bulk }}$ and negative ions $n_{-}(S) / n_{- \text {bulk. c) The correla- }}$ tion parameter $\zeta(S)$. Calculated point charges-full macro-ion solvent interface (thin solid line), $r_{\text {ion }}=0.185$ (dashes) and $r_{\text {ion }}=0.25$ (dots). Included for reference is the solution of the standard PB equation (thick solid line).

energy $\Phi(S)$ (the potential energy of a small ion in the mean field $\left.\phi_{0}(S)\right)$ calculated with $r_{\text {ion }}=0.185$. In Figure $10 \mathrm{a}$ we fix $\tilde{\kappa}=0.48$ and calculate it for various values of $\sigma_{f}$. In Figure 10b we fix $\sigma_{f}=1$ and calculate $\Phi(S)$ for various values of $\tilde{\kappa}$. The magnitude of $\Phi(S)$ increases decreasing $\tilde{\kappa}$. These qualitative features are consistent with those of point charges, where $\Phi(S)=\phi(S)$.

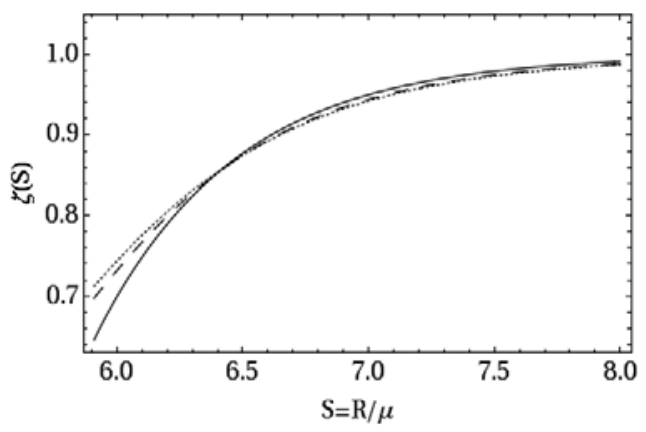

Fig. 12. Results of numerical calculations at $25 \%$ DNA charge density at $\tilde{\kappa}=0.48$ for the correlation parameter $\lambda(S)$. Calculated point charges (full macro-ion solvent interface, solid line), $r_{\text {ion }}=0.185$ (dashes) and $r_{\text {ion }}=0.25$ (dots). Included for reference is the solution of the standard PB equation (solid line).

Next, we examine what effect finite size has on various calculated functions in Figure 11. Here, we set $\sigma_{f}=0.75$ and $\tilde{\kappa}=0.48$. In general, we see that, by including finitesize effects the results become closer to those calculated for the PB equation. Most significantly in Figure 11c we see a considerable reduction in $\zeta(S)$. Correlation effects are much reduced. This is because in the close vicinity of a small ion, the electrostatic forces on other ions are very much reduced. Consequently, they are less able to screen out the electric field of such a small ion. It is this reduction in $\zeta(S)$ which is the dominant effect of including finite charge distributions. We find that for all the cases considered $\Phi(S) \approx \phi(S)$. However, this will certainly change with increasing $r_{\text {ion }}$.

In Figure 12 we examine what effect the finite size of the charge distribution on small ions has at smaller fixed surface charge densities on $\zeta(S)$, namely $\sigma_{f}=0.25$, $\tilde{\kappa}=0.48$. We see that the effects of including finite size are very much reduced. When $S$ is small we see that the effect of increasing the size of the charge distribution on the small ions is to increase $\zeta(S)$. At close distances, the ionic exclusion and image charge effects dominate. Therefore an explanation for the observed trend is that the degree of image charge repulsion an ion experiences, from the surface of the macro-ion, is smaller, the larger the size of the extended charge distribution. At larger values of $S$, the point-like ions benefit more from correlation effects, which leads to point ions having the largest value of $\zeta(S)$, but for $\sigma_{f}=0.25$ any difference is very slight.

In Figure 13 we show calculations of the charge density of the small ions. One should notice that the extended distributions penetrate into the region $\tilde{a}<S<\tilde{b}$. Indeed, this allowed for the finite charge distributions; the requirement is that the ionic centers lie outside $S=\tilde{b}$. Indeed, the tail, for small $S$ in the charge density, is due individual charge distributions of each ion at $S<\tilde{b}$ decaying away exponentially with decreasing $S$. Very little of the charge distribution penetrates into the macro-ion core, therefore an assumption (App. D) which is used in solving the modified $\mathrm{PB}$ equation for ions with extended 

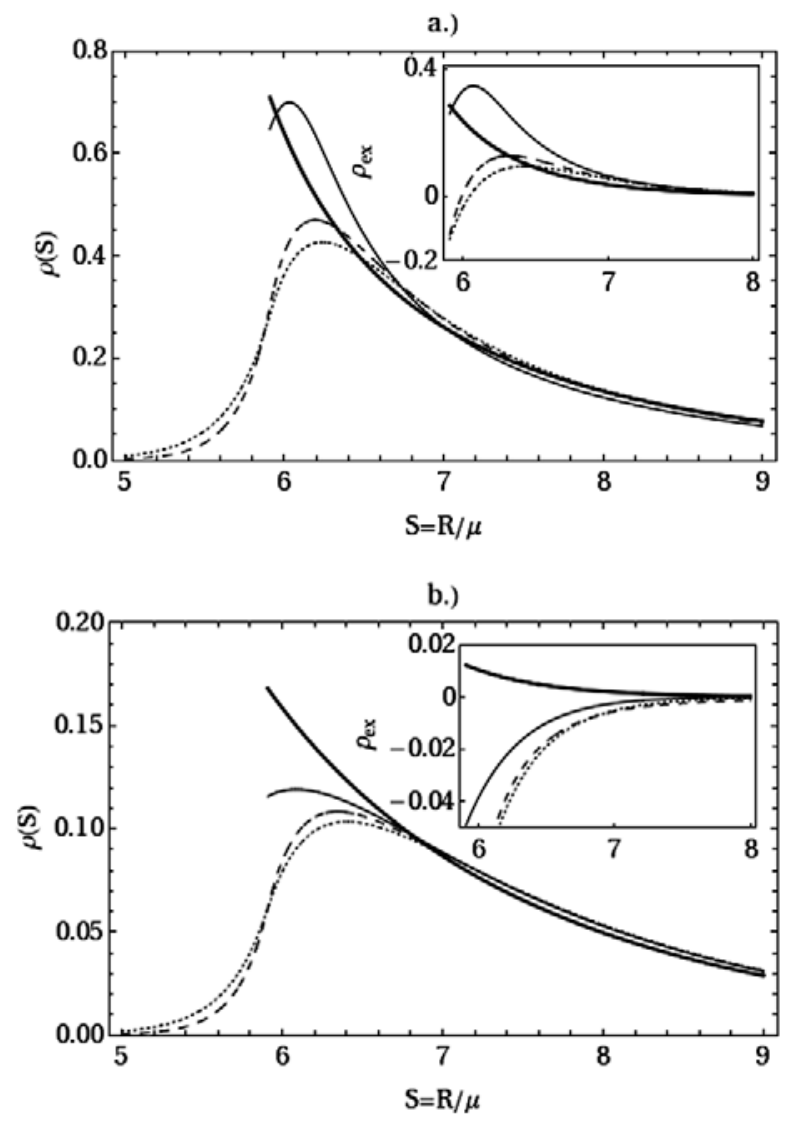

Fig. 13. Calculated full charge densities (main graph) and excess charge densities (inset) for $\tilde{\kappa}=0.48$; calculated at a) $75 \%$ and b) $25 \%$ of the DNA charge density; using $\mathrm{PB}$ equation (thick solid line), modified $\mathrm{PB}$ equation for point charges (thin solid line line), $r_{\text {ion }}=0.185$ (dashes) and $r_{\text {ion }}=0.25($ dots $)$.

charge distributions is completely justified. In the insets we also show the excess charge distribution $\rho_{\text {ex }}(S)$. For extended charge distributions the excess charge density (so that the linearized PB equation, Eq. (7.1) may be used in all cases) is given by

$$
\rho_{\mathrm{ex}}(S)=\rho_{c}(S)+\kappa^{2} \phi_{0}(S)
$$

We see that at $\sigma_{f}=0.25$, neglecting $\rho_{\text {ex }}$ may be an adequate approximation, but for $\sigma_{f}=0.75$ this approximation does not work well in all cases.

An important feature to see in the plots of $\rho_{\mathrm{ex}}(S)$, for the extended charge distributions, is that the excess goes negative near $S=\tilde{b}$. This reflects the relative inefficiency of extended ions to screen out the electrostatic interaction, when compared to point charges.

For extended charge distributions the correction $\Delta \tilde{E}_{H}(\mathbf{s}, \mathbf{s})$ from the exact equations has not been calculated. Though it is completely feasible to calculate, the calculations are a little more involved, and so have not yet been done. The corrections may be smaller due to the fact that $\zeta(S)$ is smaller.

\subsection{Effective interaction between two uniformly charged cylinders}

Understanding the distribution of counter-ions is important for calculating the interaction between macro-ions in solution. By calculating $\rho_{\mathrm{ex}}(S)$ one may calculate an effective renormalized surface charge which may be used in a DLVO approximation to describe the interaction between two such ions. Also, knowledge of $\rho_{\text {ex }}(S)$ can tell us roughly where such an approximation is likely to break down. One should point out that in such an approximation, attractive interactions from correlations are neglected. Though, for univalent ions the former term may be quite small compared to the other terms in the interaction. Utilizing the DLVO approximation for two uniformly charged cylinders, we may write down the following effective pair potential [1], here written in normal SI units of length:

$$
\frac{V_{\mathrm{int}}}{L k_{B} T}=2(1-\theta)^{2} \frac{l_{B}}{l_{c}^{2}}\left(\frac{K_{0}\left(\kappa_{D} R\right)+\Omega\left(\kappa_{D} R, \kappa_{D} a\right)}{\left(\kappa_{D} a\right)^{2}\left[K_{1}\left(\kappa_{D} a\right)\right]^{2}}\right),
$$

where

$$
\Omega(x, y)=-\sum_{j=-\infty}^{\infty} K_{j}(x)^{2} \frac{I_{j}^{\prime}(y)}{K_{j}^{\prime}(y)} .
$$

Here $l_{c}$ is the distance between two fixed charge groups on the macro-ion divided by their charge and is related to the surface charge density $l_{c}=\left(2 \pi \sigma_{s} a\right)^{-1}$. The first term in equation (7.13) represents the direct electrostatic interaction between the macro-ions, which is enhanced by the low dielectric cores by a factor of $\left(\kappa_{D} a\right)^{-2}\left[K_{1}\left(\kappa_{D} a\right) I_{0}\left(\kappa_{D} a\right)\right]^{-2}$. The second term represents an image charge repulsion term, where both the excess counter-ions and fixed charges are repelled by their image charges on the surface of the other macro-ion.

A possible criteria for equation (7.13) being an adequate description of the repulsive part of the interaction is that the excess charge distributions, $\rho_{\text {ex }}(S)$ of the two macro-ions do not overlap to a great extent. However, when $\theta \simeq 1$, equation (7.13) should always work for a local dielectric description of the solvent. On examination of the excess charge densities in Figure 12, we see that, at an inter-axial separation of $R \gtrsim 14(30 \AA)$ (for charge density $\sigma_{f}=0.75$ in a salt solution with $\tilde{\kappa}=0.48$, Debye screening length $\lambda \simeq 4.58 \AA$ ) this criterium is roughly satisfied.

From $\rho_{\text {ex }}(S)$ we are able to calculate $\theta$ through the following expression (in rescaled units):

$$
\begin{aligned}
& \theta= \\
& \frac{\tilde{k}}{2 \sigma_{f}}\left(\int_{\tilde{b}}^{\infty} S \mathrm{~d} S\left[I_{0}(\tilde{\kappa} S) K_{1}(\tilde{\kappa} \tilde{a})+I_{1}(\tilde{\kappa} \tilde{a}) K_{0}(\tilde{\kappa} S)\right] \rho_{\mathrm{ex}}(S)\right. \\
& +\int_{\tilde{a}}^{\tilde{b}} S \mathrm{~d} S\left(\rho_{c}(S)+\kappa^{2} \phi_{0}(S)\right)\left[I_{0}(\tilde{\kappa} S) K_{1}(\tilde{\kappa} \tilde{a})\right. \\
& \left.\left.+I_{1}(\tilde{\kappa} a) K_{0}(\tilde{\kappa} S)\right]\right)
\end{aligned}
$$


Table 1. Calculated values of $\theta$ at $\tilde{\kappa}=0.32\left(\kappa_{D} \approx 0.14 \AA^{-1}\right)$ for the various sizes of ions used in the modified PB equations as well as the PB equation.

\begin{tabular}{|l|l|l|c|c|c|}
\hline$\sigma_{f}$ value & PB equation & $\begin{array}{l}\text { Point charges } \\
\text { scenario 3) }\end{array}$ & $\begin{array}{c}\text { Point charges } \\
\text { scenario 4) }\end{array}$ & $\begin{array}{c}\text { Full theory } \\
r_{\text {ion }}=0.185\end{array}$ & $\begin{array}{c}\text { Full theory } \\
r_{\text {ion }}=0.25\end{array}$ \\
\hline 0.25 & $\theta=-0.350(0.044)$ & -0.339 & -0.404 & -0.380 & -0.391 \\
\hline 0.5 & $-0.206(0.146)$ & -0.0755 & -0.120 & -0.136 & -0.132 \\
\hline 0.75 & $-0.0205(0.280)$ & 0.186 & 0.157 & 0.100 & 0.0921 \\
\hline 1 & $0.134(0.388)$ & 0.406 & 0.386 & 0.288 & 0.275 \\
\hline
\end{tabular}

Table 2. Calculated values of $\theta$ at $\tilde{\kappa}=0.48\left(\kappa_{D} \approx 0.22 \AA^{-1}\right)$ for the various sizes of ions used in the modified PB equations as well as the PB equation. Here N.S. means that no solution was obtained.

\begin{tabular}{|l|c|c|c|c|c|}
\hline$\sigma_{f}$ value & PB equation & $\begin{array}{c}\text { Point charges } \\
\text { scenario 3) }\end{array}$ & $\begin{array}{c}\text { Point charges } \\
\text { scenario 4) }\end{array}$ & $r_{\text {ion }}=0.185$ & $r_{\text {ion }}=0.25$ \\
\hline 0.25 & $\theta=-0.696(0.032)$ & -0.748 & -0.842 & -0.776 & -0.659 \\
\hline 0.5 & $-0.581(0.108)$ & -0.473 & -0.542 & -0.550 & -0.537 \\
\hline 0.75 & $-0.435(0.213)$ & -0.167 & -0.215 & -0.299 & -0.304 \\
\hline 1 & $-0.285(0.315)$ & N.S. & N.S. & -0.0950 & -0.0951 \\
\hline
\end{tabular}

This equation has been modified from the one originally considered in [53], by a second term that takes into account that no charge centers can lie at $R<\tilde{b}$, and any counter-ion charge distribution, $\rho_{c}(S)$ that lies within this region due to extended charges. In Tables 1 and 2 we show the calculated values for the charge compensation parameter for $\tilde{\kappa}=0.32$ and $\tilde{\kappa}=0.48$, respectively.

For $\sigma_{f}=0.25$ we see that the values are quite negative, most of this comes from the fact that ions are excluded a distance $b-a$ away from the core region. Through the second term in equation (7.15), this leads to an enhancement of the surface charge; the ions less effectively screen out the surface charge due to this. For illustration, in brackets, are the values calculated with the $\mathrm{PB}$ equation not taking into account the second term of equation (7.15). First from the results for point charges, we see that image and exclusion effects, in the self-energy, lower $\theta$. Furthermore, we see that finite size reduces these effects. This is consistent with what is seen in Figure 12 for $\rho_{\mathrm{ex}}(S)$. The values are lower for $\tilde{\kappa}=0.48$ than for $\tilde{\kappa}=0.32$, where the second term of equation (7.15) is largest in magnitude. Decreasing the screening length increases the amount of charge compensation in all cases.

As is expected, as one increases $\sigma_{f}$ the charge compensation increases. Also, we see that as we increase $\sigma_{f}$, including image charges seems to matter less; ionic exclusion effects matter more. For $\sigma_{f}=0.75$ the modified PB equation for point ions in scenario 3 ), no image charges, gives the largest value of the compensation. The enhancement of the compensation, above the values calculated for just the PB equation, is due to the correlation effects [46], which, as we have seen, increases the surface density of ions. These compensation values decrease as the more spread out the distribution of charge on the small ions becomes; there is a significant reduction in $\theta$ for values of $r_{\text {ion }}$ considered, when compared to point charges.
As yet, we still have to calculate an attractive term for correlation effects, as well as accounting for adjustment of ions in the charge compensation; in a later study these will be included. We certainly expect the latter to have an effect on the DNA-DNA interaction as the cylinders are brought closer together.

\section{Discussion}

In the numerical solution of the simplified equations, so far, only univalent ions have been considered. It is possible to consider divalent ions, where the effects are likely to become much more pronounced. But care here should be taken with the WKB-like approximation. It may only work well, here, for relatively small surface charge densities, compared to DNA. Indeed, it is already seen to breakdown for univalent ions at the DNA charge surface density. Perhaps, to get a better approximation, without having to solve the full Hartree equations or include the correction, it might be possible to use the WKB-like form for the correlation function as variational ansatz. Here, $\hat{\kappa}(\mathbf{s}, 0)$ would now be treated as a variational parameter along with $\phi_{0}(\mathbf{s})$, on the substitution of $\tilde{V}_{H}\left(\mathbf{s}, \mathbf{s}^{\prime}\right)$ (full expression in App. C) into equation (5.4). Such a variational approximation could also be used for extended distributions. However, this still remains an intriguing possibility that has yet to be explored. Nevertheless, as the valance of ions is increased, we expect the Hartree equations and the whole variational approach to work less well. For univalent ions for the charge densities explored, the counter-ion distributions and the electrostatic potential lie relatively close to the saddle point approximation, since $\zeta(S) \approx 1$. Therefore we suppose that the Hartree approximation should be relatively accurate as it explicitly includes the first-order perturbation result, but this can be tested through higher- 
order perturbation theory, more sophisticated approximations and simulation. But certainly, for trivalent point-like ions, we expect the Hartree approximation to breakdown, close to the surface of the macro-ion. Near the surface, a strong-coupling regime [3] will hold, as $\Xi$ is so high. Yet, the situation may be a little more subtle for real ions with valences larger than, or equal to, three. These really cannot be considered to be point-like $[1,54]$. And as we have seen in this study that, for extended distributions, the correlation effects can reduce quite markedly.

The main connection of this work, with experiment, is determining the strength interaction between molecules. Notably, these forces have been measured in assembly for DNA [55]. Indeed, calculating the charge compensation parameter $\theta$ which renormalizes the surface charges in a DLVO approximation for the interaction between two cylinders is an important step in that direction. However, one should point out that there have also been attempts to measure the profile of the counter-ion atmosphere directly through X-ray diffraction [56], though such experiments were at the limit of experimental accuracy [1].

Indeed, image charge effects, and the exclusion of ions from the core of the macro-ion, push down the value of $\theta$ and correlation effects push it up. Also, by not allowing the charge centers of ions to come to the surface of the dielectric core (i.e. requiring $b>a$, where $a$ and $b$ are the radius of the dielectric core and the minimum approach radius of an ion, respectively), we also cause a considerable reduction in $\theta$, which actually leads to an enhanced renormalized charge. Also, with increasing $\sigma_{f}$ the inclusion of image charges seems to matter less; only at intermediate values of $\sigma_{f}=0.5,0.75$ are they significant. With our results we are able to make estimates of below what separation the DLVO approximation is likely to break down. However, in the case of a molecule like DNA, such estimates may be too conservative for two reasons. The first is that DNA is not a smooth cylinder; it has grooves in which ions may sit accommodating their hydration shells. The second is that chemi-adsorption is important for many species of ion, even mono-valent alkali metals (for example $\mathrm{Na}^{+}$) feel the presence of fractional charges on the base pairs within the grooves [1]. Chemi-adsorption will bring more of the condensed ions, those contributing to $\theta$, closer to the macro-ion surface, and this in turn should reduce the separation above which this effective (repulsive) interaction is valid. Of course the full effective interaction has yet to include an attractive term due to correlation effects as well as counter-ion adjustment, thus going beyond the DLVO approximation.

Chemi-adsorption is a missing ingredient that has yet to be included [57] consistently in the theory. This cannot simply be included in just the equation for the electrostatic field; it affects ionic correlations as well. This is because ions adsorbed at the surface may be able to adjust themselves to the presence of an ion out in the solution, so creating a correlation hole. This effect should draw small ions closer, pushing up $\theta$. Though, this effect may depend heavily on where the ions are adsorbed and spatial characteristics of the adsorption potential. To study this effect, we hope to include chemi-adsorption in a modified WKB- like approximation and its interplay with correlation and image effects in future work.

A second missing ingredient is a full treatment of the finite-size effects of the small ions. Already, we have looked at the finite size of the charge distribution of ions, which seems very important for the correlation effects. Also, we have considered a minimum closest approach for an ion. However, steric/short-range potentials should be included consistently in the statistical-mechanical model. In the simplest approximation, each ion may be treated as a hard sphere, but not just at the surface of the macro-ion, in the solution as well. The hard-core radius of each ion in solution may be taken to be that of the ion and a tightly bound first hydration shell of water. We are currently developing techniques to account for this in the field-theoretical approach. The goal being to develop a type of equation similar to that considered in [42], modified to take account of weak correlation effects. However, we want develop this from a more rigorous statistical mechanical approach, for the macro-ion-electrolyte system, than a lattice gas. This equation should also include both correlation and image charge effects (through coupling to an equation describing the correlation function).

Of course, simulations to test at what values of the correlation strength $\Xi$, and other parameters, the Hartree approximation works would be useful. Also, one could also try and test this by computing the next-to-leading order correction of the full theory to the variational trial functional or by trying to develop a random phase approximation. But, to go beyond the Hartree equations full steric effects are essential. One can show that this correction to the Hartree approximation, for point charges without steric effects, is highly divergent. This is a manifestation of the Bjerrum instability towards the formation of Bjerrum pairs. Therefore, to calculate a meaningful correction, steric effects are essential; the hard-core size of the ion acts as a cutoff. Indeed, there should be a very important interplay between effective size of ions and the validity of the Hartree approximation on its own. If the hard-core radius of an ion is too small, then the Hartree approximation will not work and a large proportion of ions will form electrically neutral bound Bjerrum pairs. These considerations should also be important in looking for a strong-coupling expansion $[3,10]$ in the presence of salt.

Of course, our description solvent may be too simple: a constant bulk dielectric. So another direction of development would be to consider modeling the solvent in a more sophisticated way. This might be achieved through two possible routes. The first is to treat the solvent explicitly as individual dipoles. It is certainly possible to include the solvent explicitly in the field theory of a single fluctuating field [18]. The problem with this approach, in water (or other strong polar solvents), is that the dipoles interact strongly (highly correlated), so one would have to go very much beyond the mean-field approach. Finite-size steric effects of the water molecules would also need to be considered. Though, solving the simpler problem of ions in a dilute weak polar solvent may still be insightful [18]. A second approach, more phenomenological, would be to 
couple the counter-ions to a Landau-Ginzburg model, describing a local polarizability field $[47,17]$. Such a model has enjoyed some success in describing the microscopic electrostatic effects of water [47]. Whatever the approach used, this course of study is likely to be very involved, and should be left until later in the development of the theory.

\section{Conclusion and outlook}

In this work we have developed a field-theoretic formalism to handle three effects that go beyond the simple PB approach: image charge effects of the small ions, weak correlation effects, and finite ionic charge distributions. From this field theory we have derived self-consistent (Hartree) equations; a modified PB equation and an equation for the correlation function of a fluctuating field, which describes the additional correlation effects and image charge effects. These equations may apply to any solution of small rigid ions with extended charge distributions, most notably cobalt hexamine and putracine.

As a first attempt at obtaining approximate solutions to these equations we have developed a WKB-like approximation. We have used this approximation to calculate the mean electrostatic potential and distribution of small ions about a uniform distribution of fixed charges on a cylindrical macro-ion. We have done this for point charges and spherically symmetric charge distributions of small univalent ions.

For point charges, we find that ionic exclusion effects cause a considerable reduction in $\zeta(S)$. When we include image charges, there is a further smaller, though significant reduction. Whereas, correlation effects have the opposite effect; pushing up $\zeta(S)$. When the surface charge is low, the local concentration of ions is dominated by exclusion effects and image charge repulsion, and so diminishes as we move close to the surface of the macro-ion. As we increase the macro-ion charge density, we move into a regime where correlation effects win out over image charge effects and the local concentration of ions increases slightly more than what the $\mathrm{PB}$ equation predicts. Also, by considering a correction, we have shown that WKB-like approximation works well compared to full Hartree result for point ions.

When we make the charge distribution on the small ions finite, we find that, for values of the surface charge density close to DNA, $\zeta(S)$ is very much reduced and lies much closer to one; the results are closer to the PB equation. At surface charge densities considerably smaller than DNA, $\zeta(S)$ changes little for the sizes of charge distribution considered.

The main application of all this work is the in the theory of interaction between macro-ions. Here, we have considered the interaction of two cylinders in the DLVO approximation. From our calculations it possible to calculate a factor $1-\theta$ that renormalizes the fixed surface charges density in such an approximation. We find that correlations, image charges and finite size all have a significant effect on $\theta$, which becomes important when the surface charge density is comparable to that of DNA. The upshot is that all of these effects could be important in the interaction, though the effects seem to cancel each other out to a certain degree.

In following publications we hope to investigate chemiadsorption and the effect of including steric interactions between ions. Also, it will be interesting to apply what is learnt to distributions of helical charge. Here, from our microscopic theory, we would want to calculate the helical moments (KL parameters) presented in [1], these are important for the interaction between helical macro-ions. Finally, we will want to consider the Hartree approximation of a system of two macro-ions. Our goal is to treat the interaction between helical macro-ions in a more consistent way than in [12]. Then we may examine the validity of the effective KL theory for helical molecules [1,12] and, where appropriate, modify the KL theory to take account of correlation effects and counter-ion readjustment.

D.J. Lee would like to acknowledge the support of the MaxPlanck Institute for the Physics of Complex Systems and A.A. Kornyshev for useful discussions.

\section{References}

1. A.A Kornyshev, D.J. Lee, S. Leikin, A. Wynveen, Rev. Mod. Phys. 79, 943 (2007), and references therein.

2. A. Naji, A. Arnold, C. Holm, R.R. Netz, Europhys. Lett. 67, 130 (2004).

3. A. Naji, R.R. Netz, Eur. Phys. J. E 13, 43 (2004).

4. R.E. Goldestanian, T.B. Liverpool, Phys. Rev. E 66, 051802 (2002).

5. A. Naji, S. Jungblut, A.G. Moreia, R.R. Netz, Physica A 352, 132 (2005), and references therein.

6. A.W.C. Lau, P. Pincus, D. Levine, H.A. Fertig, Phys Rev. E 63, 051604 (2001).

7. A. Grosberg, T. Yu, T. Nguyen, B.I. Shklovskii, Rev. Mod. Phys. 74, 329 (2002), and references therein.

8. P. Grochowski, J. Trylska, Biopolymers 89, 93 (2007), and references therein.

9. M. Deserno, M.A. Arnold, C. Holm, Macromolecules 36, 249 (2003).

10. M. Kandǔc, R. Podgornik, Eur. Phys. J. E. 23, 265 (2007).

11. Y. Levin, Rep. Prog. Phys. 65, 1577 (2002), and references therein.

12. A.A Kornyshev, S. Leikin, J. Chem. Phys. 107, 3656 (1997).

13. R. Podgornik, B. Žekš, J. Chem Soc., Faraday Trans. 2, 5, 611 (1988).

14. S. Marčelja, N. Radic, Chem. Phys. Lett. 42, 129 (1976).

15. O.V. Dolgov, D.A. Kirhnitz, E.G. Maximov, Rev. Mod. Phys. 53, 81 (1981).

16. A.A Kornyshev, in The Chemical Physics of Solvation, edited by R.R. Dogonadze, E. Kalman, A.A. Kornyshev, J. Ulstrup (Elsevier, Amsterdam, 1985).

17. I.G. Medvedev, Rus. J. Elctrochem. 37, 193 (2001).

18. A. Abrashkin, D. Andelman, H. Orland, Phys. Rev. Lett. 99, 077801 (2007).

19. R. Kjellander, Colloid J. 69, 20 (2007).

20. A.A Kornyshev, S. Leikin, Phys. Rev. Lett. 82, 4138 (1999).

21. Z.J. Lian, H.R. Ma, J. Chem. Phys. 127, 104507 (2007).

22. A.G. Moreira, R.R. Netz, Europhys. Lett. 57, 911 (2002). 
23. E. Raspuad, I. Chperon, A. Leforestier, F. Livolant, Biophys. J. 77, 1547 (1999).

24. M.T. Saminathan, A. Antony, A. Shirahata, L.H. Sigal, T. Thomas, T.J. Thomas, Biochemistry 38, 3821 (1999).

25. I. Rouzina, V.A. Bloomfield, J. Phys. Chem. 100, 9977 (1996).

26. A.A. Kornyshev, D.J. Lee, S. Leikin, A. Wynveen, S.B. Zimmerman, Phys. Rev. Lett. 95, 148102 (2005).

27. J. Ruiz-Chica, M.A. Medina, F. Sánchez-Jiménez, F.J. Ramirez, Biophys. J. 80, 443 (2001).

28. A.A. Ouameur, H.A. Tajmur-Riahi, J. Biol. Chem. 279, 42041 (2004).

29. C.A. Davey, T.J. Richmond, Proc. Natl. Acad. Sci. U.S.A. 99, 11169 (2002).

30. E. Moldrheim, B. Anderson, N.A. Froystein, E. Sletten, Inorg. Chim. Acta 273, 41 (1998).

31. A.A. Kornyshev, S. Leikin, Phys. Rev. E. 62, 2576 (2000).

32. A.A. Kornyshev, S. Leikin, S.V. Malinin, Eur. Phys. J. E 7, 83 (2002).

33. A. Wynveen, D.J. Lee, A.A. Kornyshev, Eur. Phys. J. E 16, $303(2005)$.

34. D.J. Lee, A. Wynveen, J. Phys. Condens. Matter 18, 787 (2006).

35. A.G. Cherstvy, A.A. Kornyshev, S. Leikin, J. Phys. Chem B 108, 6508 (2004).

36. D.J. Lee, A. Wynveen, A.A. Kornyshev, Phys. Rev. E 70, 051913 (2004).

37. A. Wynveen, D.J. Lee, A.A. Kornyshev, S. Leikin, Nucl. Acids Res. 36, 5540 (2008); in preparation.

38. T.J. Grycuk, Phys. Chem. B 106, 1434 (2002).

39. D.C. Rau, V.A. Parsegian, Biophys. J. 61, 246 (1992).

40. A. Wynveen, F. Bresme, J. Chem. Phys. 124, 104502 (2006).

41. F. Bresme, A. Wynveen, J. Chem. Phys. 126, 044501 (2007).

42. I. Borukhov, D. Amdelman, H. Orland, Electrochim. Acta 46, $221(2000)$.

43. R.R. Netz, H. Orland, Eur. Phys. J. E 1, 203 (2000).

44. S.L. Carnie, G.M. Torre, Advances in Chemical Physics, edited by L. Prigogine, S.A. Rice, Vol. 56 (Wiley, New York, 1984).

45. D.J. Amit, Field Theory, the Renormalization Group, and Critical Phenomena (World Scientific, London, 1989).
46. This mechanism has been discussed in the case of a Wigner crystal (A. Grosberg, T.Yu, T. Nguyen, B.I. Shklovskii, Rev. Mod. Phys. 74, 329 (2002)), and in such an extreme case may lead to overcharging.

47. It might be better to consider finite-size effects in this term using some modifications of the Born approximation, to take into account the fact that other ions and solvent molecules cannot penetrate into the ion. Therefore, possibly a better choice of $\tilde{f}\left(\mathbf{s}^{\prime}-\mathbf{s}\right)$ might be to consider an effective charge distribution of a uniformly charged shell (or a smeared charged shell) with the same radius as that of the ion. For instance, see A.A. Kornyshev, G. Sutmann, J. Electroanal. Chem. 450, 143 (1998). However, the resulting equations are much harder to work with, and so we have not considered this.

48. The correlation parameter appears much larger than what it would be in R.R. Netz, H. Orland, Eur. Phys. J. E 11, 301 (2003) $(\Xi \approx 3)$, due to the difference of a factor of $2 \pi$ in the definition.

49. Here we should remind the reader that $S=R / \mu$, where $R$ is the standard radial coordinate in polar coordinates measured in S.I. units, and the major axis of the macroion is taken to coincide with the $z$-axis.

50. R.J.F. Leote de Carvalho, E. Trizac, J.P. Hansen, Phys. Rev. E 61, 1634 (2000).

51. In particular, the value $r_{\text {ion }}=0.185$ was chosen due to $\sqrt{\left\langle r^{2}\right\rangle_{f}}=1 \AA$ (for $\mu=2.2 \AA$ ), the size of a typical ion, where $\langle g(\mathbf{s})\rangle_{f}=\int \mathrm{d}^{3} s f(\mathbf{s}) g(\mathbf{s})$ and $f(\mathbf{s})$ is defined through equation (6.7). Though, strictly, care should taken in relating $r_{\text {ion }}$ to the ion size.

52. It is probable that such "over-screening" phenomena could very much depend on the choice of the charge distribution and may require careful investigation.

53. EPAPS Document No. E-RMPHAT-79-010702.

54. For spermine and other polyamides, the physics could be quite complicated as spermine forms a flexible rod along which four unit positive charges are equally distributed. To take account of the flexibility requires more degrees of freedom to be assigned to each molecule.

55. D.C. Rau, V.A. Parsegian, Biophys. J. 61, 246; 260 (1992).

56. R. Das, T.T. Mills, L.W. Kwok, K.D. Finkelstein, D. Herschlag, L. Pollack, Phys. Rev. Lett. 90, 188103 (2003).

57. In preparation. 